\title{
Ligand Binding-Dependent Functions of the Lipocalin NLaz: an in vivo Study in Drosophila.
}

\author{
Mario RUIZ ${ }^{1}$, Maria D. GANFORNINA ${ }^{1 *}$, Colin CORRENTI ${ }^{2}$, Roland K. STRONG ${ }^{2}$ \\ and Diego SANCHEZ ${ }^{*}$, \\ ${ }^{1}$ Instituto de Biología y Genética Molecular-Departamento de Bioquímica y Biología \\ Molecular y Fisiología, Universidad de Valladolid-CSIC, 47003, Valladolid, Spain. \\ ${ }^{2}$ Division of Basic Sciences, Fred Hutchinson Cancer Research Center, Seattle, WA \\ 98109, USA \\ * MDG and DS contributed equally to this work.
}

Running title: In vivo test of NLaz lipid binding-related roles

Abstract word count: 181

Introduction word count: 763

Materials and Methods word count: 1060

Results word count: 2380

Discussion word count: 880

Bibliography: 69 references, 2299 words

Legends word count: 1088

Total word count: 8651

Number of figures: 5

Number of tables: 2

Number of Supplementary figures: 2

Number of Supplementary data files: 2

\author{
${ }^{\S}$ Author for correspondence: \\ Diego Sanchez \\ Instituto de Biología y Genética Molecular, \\ c/ Sanz y Forés 3, \\ Universidad de Valladolid-CSIC, \\ 47003 Valladolid, Spain. \\ Phone: 983-184814 \\ Fax: 983-184800 \\ e-mail: 1azarill@ibgm.uva.es
}

\section{Contact information for all other authors:}

Mario Ruiz maritoruiz@gmail.com

Maria D Ganforninaopabinia@ibgm.uva.es

Colin Correnti ccorrent@,fhcrc.org

Roland K. Strong rstrong@fhcrc.org 


\section{LIST OF NON-STANDARD ABBREVIATIONS}

AGE: Advanced glycation end products

ApoD: Apolipoprotein D

BSA: Bovine serum albumin

CNS: Central nervous system

da: daughterless

DMF: N,N-dimethylformamide

ECL: Enhanced chemiluminescence

EDTA: Ethylenediaminetetraacetic acid

FOXO: Forkhead box O1 protein

GLaz: Glial Lazarillo

GPI: Glycosylphosphatidylinositol

hApoD: human Apolipoprotein D

HRP: Horseradish peroxidase

IgG: Immunoglobulin $\mathrm{G}$

IIS: Insulin/IGF signaling (IGF: insulin-like growth factor)

JNK: Jun-N-terminal Kinase

$K_{d}$ : Dissociation constant

Len2: lipocalin 2

L-PGDS: lipocalin type - Prostaglandin D synthase

NGAL: Neutrophil gelatinase-associated lipocalin

NLaz: Neural Lazarillo

NLaz-KO: Neural Lazarillo knock-out mutant

PNGase-F: peptide-N-glycosidase F

PQ: Paraquat

RA: retinoic acid

RBP: Retinol Binding Protein

RpL32: Ribosomal protein L32

SDS-PAGE: sodium docecyl sulphate - polyacrylamide gel electrophoresis

TI: Trypsin inhibitor

TOR: Target of rapamycin protein

UAS: Upstream activated sequence

WT: wild type 


\begin{abstract}
Lipocalins are small extracellular proteins mostly described as lipid carriers. The Drosophila lipocalin NLaz (Neural Lazarillo) modulates the IIS pathway and regulates longevity, stress resistance, and behavior. Here we test whether a native hydrophobic pocket structure is required for NLaz to perform its functions. We use a point mutation altering the binding pocket $\left(\mathrm{NLaz}^{\mathrm{L} 130 \mathrm{R}}\right)$ and control mutations outside NLaz binding pocket. Tryptophan fluorescence titration reveals that $\mathrm{NLaz}^{\mathrm{L} 130 \mathrm{R}}$ loses its ability to bind ergosterol and the pheromone 7(z)-tricosene, but retains retinoic acid binding. Using site-directed transgenesis in Drosophila we test the functionality of the ligand binding-altered lipocalin at the organism level. NLaz-dependent lifespan reduction, oxidative stress and starvation sensitivity, aging markers accumulation, and deficient courtship are rescued by overexpression of $\mathrm{NLaz}^{\mathrm{WT}}$, but not of $\mathrm{NLaz}^{\mathrm{L} 130 \mathrm{R}}$. Transcriptional responses to aging and oxidative stress show a large set of ageresponsive genes dependent on the integrity of NLaz binding pocket. Inhibition of IIS activity and modulation of oxidative stress and infection-responsive genes are binding pocket-dependent processes. Control of energy metabolites upon starvation appears to be, however, insensitive to the modification of the NLaz binding pocket.
\end{abstract}

\title{
Keywords:
}

Aging

Oxidative stress

Lipid-binding proteins

Pheromonal signalling

Metabolism regulation 


\section{INTRODUCTION}

Lipocalins are a large family of small extracellular proteins characterized by their well conserved $\beta$-barrel structure embracing an internal cavity able to bind small ligands, most of them hydrophobic or amphipathic (1). For some lipocalins we have a good approximation to the role played by their native ligand. For instance, Retinol Binding Protein (RBP) transports vitamin A within the retina (2); Siderocalin (Lcn2 or NGAL) sequesters iron-binding molecules and provides a bacteriostatic mechanism against infections (3); and Crustacyanins carry the pigment giving color to the carapace of crustaceans (4). However the panoply of different physiological functions in which lipocalins are involved, many of them tested by altering their expression levels in genetically-modified organisms (5-8), keeps increasing, and in the vast majority of cases a ligand transport function is assumed but not tested.

A set of lipocalins, including the most ancient ones within the metazoan lineage (911 ), has been functionally linked to the response of organisms to different forms of stress and to the modulation of metabolism, key parameters regulating senescence and longevity (reviewed by 12). Among these are the Lazarillo-related lipocalins, a group including the Drosophila lipocalins Neural Lazarillo (NLaz) and Glial Lazarillo (GLaz) (13) and the vertebrate Apolipoprotein D (ApoD). The expression of these lipocalins, both within and outside the nervous system, is boosted by oxidative or metabolic stress and they provide an endogenous mechanism of protection particularly important in situations of aging and neurodegeneration $(5,14-20)$.

NLaz is induced under the control of the stress responsive JNK pathway, and is able to negatively regulate the IIS pathway $(16,21)$. NLaz expression increases stress resistance and longevity, but also serves other apparently unrelated functions by regulating fecundity, food-intake and locomotor and courtship behaviors $(18,22)$. The molecular mechanism by which NLaz is able to perform these varied functions has not been elucidated yet, although general proposals of lipid-transport or interaction with cellular lipid membrane surfaces have been laid out $(23,24)$. Whether all NLaz functions are carried out using a common biochemical mechanism, or this lipocalin can exert different functions depending on the ligand available for binding in different physiological situations, is still unknown.

Studies testing ligand binding abilities of lipocalins in vitro are abundant (25-27), including ApoD and Lazarillo-related lipocalins (23, 28-32). In addition, many lipocalins have been extensively studied at the molecular level, revealing particular residues and regions of the protein scaffold that are important for their ligand binding properties $(25,27,33-36)$. The biochemical and biophysical properties of lipocalinligand interactions have been reported for some family members, either by solving their crystal structure with the ligand bound to the pocket $(31,37)$ or by site-directed mutagenesis of residues contributing to the pocket interactive surfaces $(26,27,35$ 41). Amino acid substitutions that naturally occur in the binding pocket of RBP result in vitamin A deficiency causing night blindness (42). Also, thanks to their very robust fold, the lipocalins have been used as starting point for protein engineering. Using site-directed random mutagenesis followed by selection techniques, an increasing set of so-called "Anticalins" has been generated with various specific target binding activities, many of them with potential therapeutic benefits (for a review see 43). 
In spite of the many known lipocalin mutant forms that result in deficient ligand binding in vitro, studies analyzing their effects outside the test tube are rare. Mutant forms of Prostaglandin D synthase (L-PGDS) have recently been tested for their ability to promote cell survival in $\mathrm{H}_{2} \mathrm{O}_{2}$ treated neuronal cultures (44). A mechanism involving the titration of a free thiol in the protein without alterations of ligand binding capacity has been found. Modifications of the binding pocket of the lipocalin C8 $\gamma$ have been tested for hemolytic activity (35). Also, Correnti et al. (39) have found that Siderocalin binding pocket mutants that cannot bind siderophores in vitro lose their bacteriostatic activity when added to bacterial cultures.

The purpose of this study is to test whether the functions of the lipocalin NLaz are dependent on ligand binding. We designed different mutant forms of NLaz and tested them at three levels of analysis. We assayed their ligand binding capacity in vitro, their cell survival promoting activity in a cell culture system, and their effect on longevity, stress resistance, behavior and downstream transcriptional responses in vivo. By using site-directed transgenesis in Drosophila, we were able to compare the physiological effects of wild type and mutant NLaz expression without the confounding effect of transgene insertion site in the genome. This is, to our knowledge, the first in vivo test of the physiological outcome derived from a ligand binding-altered lipocalin at the organism level.

\section{MATERIALS AND METHODS}

\section{Homology modeling of NLaz 3D structure.}

The NLaz model was obtained from the Swiss Model Repository platform $(45,46)$, built using the atomic coordinates of hApoD [PDB entry: 2HZQ] as the best template (31). An estimation of the model quality was assessed by the QMEAN Server (http://swissmodel.expasy.org/qmean/cgi/index.cgi) (47).

\section{Cloning, S2 cell lines generation, and stress assays.}

NLaz cDNA, translating into residues 1-224 (CG33126, Uniprot reference Q9NAZ4, FlyBase entry FBgn0053126), was subcloned with a C-terminal 6xHis tail into the $\mathrm{pRmHa} 3$ vector as previously described (23). $\mathrm{NLaz}^{\mathrm{L} 130 \mathrm{R}}-\mathrm{pRmHa} 3$ was generated by direct mutagenesis of NLazWT-pRmHa3 following the Quick Change Site Direct Mutagenesis method (QIAgen). Table 1 lists the oligonucleotides used for mutagenesis, cloning, and sequencing. Transfected cells were selected with $25 \mu \mathrm{g} / \mathrm{ml}$ blasticidin-S for 3 weeks (Invitrogen).

S2 cells seeded at a density of $2 \times 10^{6} / \mathrm{ml}$ were treated with $\mathrm{H}_{2} \mathrm{O}_{2}$ as an oxidant agent in the presence of pure NLaz ${ }^{\mathrm{WT}}, \mathrm{NLaz}^{\mathrm{L} 130 \mathrm{R}}$ (see below) or a control protein (trypsin inhibitor from soybean, Sigma) as previously described (22). Viability was scored in quadruplicate wells with at least two measurements in each culture well.

\section{Purification of NLaz ${ }^{\mathrm{WT}}$ and NLaz ${ }^{\mathrm{L} 130 \mathrm{R}}$ proteins.}

Protein expression was induced in the stably transfected S2 cell lines and purified by metal-affinity chromatography followed by two rounds of size-exclusion chromatography (Supplementary Figure 1a), as previously described (23). The purified proteins were deglycosylated by treatment with peptide-N-glycosidase $\mathrm{F}$ (PNGase-F) from Flavobacterium meningosepticum (New England Biolabs) after denaturation following the protocol supplied by the manufacturer. The purified proteins were analyzed by 12\% SDS-PAGE (Supplementary Figure 1b). 


\section{Ligand binding assays by tryptophan fluorescence titration}

Fluorescence measurement were conducted as described (23) with either NLaz ${ }^{\text {WT }}$ and $\mathrm{NLaz}^{\mathrm{L} 130 \mathrm{R}}$ diluted at $1 \mu \mathrm{M}$ in $10 \mathrm{mM}$ phosphate buffer, $150 \mathrm{mM} \mathrm{NaCl}, 1 \mathrm{mM}$ EDTA at pH 7.0. The ligands tested were Retinoic Acid (Sigma), Ergosterol (Sigma) and 7(z)-Tricosene (Cayman), and were dissolved in N,N-dimethylformamide (DMF) (Sigma). An apparent $K_{d}$ was estimated with the assumption of a single binding site.

\section{Generation of transgenic flies by site-specific transgenesis.}

NLaz loss-of-function mutant (NLaz-KO) was generated in a $w^{1118}$ background (48) and crossed with a $w^{1118}$-CS wild type (WT) line to generate the NW5 line used in this study (16).

The full wild type and L130R NLaz cDNAs were subcloned into the pUASt-attB vector (49) (Gen Bank entry EF362409 and FlyBase entry FBmc0003002). A Cterminal truncated version of NLaz was generated by amplifying a cDNA fragment translating into residues 1-176 of NLaz. NLaz ${ }^{\mathrm{N} 165 \mathrm{~S}}$ was identified by sequencing as a polymorphism in some of our wild type fly stocks. These NLaz versions were also subcloned into the pUASt-attB vector. Constructs were sequenced to confirm orientation and the absence of undesirable mutations. See Table 1 for oligonucleotides used for mutagenesis and cloning.

PhiC31 system-mediated (49) transformation of Drosophila strain " $\mathrm{y}^{1} \mathrm{M}$ \{vasint.Dm $\} \mathrm{ZH}-2 \mathrm{~A} \mathrm{w}^{*}$; M $\{3 \times \mathrm{xP} 3-\mathrm{RFP}$. attP $\} \mathrm{ZH}-86 \mathrm{Fb}$ " (BL4749) was used to generate fly lines with all the transgenes inserted in the same chromosomal position $(86 \mathrm{Fb})$. NLaz transcription levels in the transgenic flies expressing $\mathrm{NLaz}^{\mathrm{WT}}$ or $\mathrm{NLaz}^{\mathrm{L} 130 \mathrm{R}}$ was compared using primers able to amplify both forms, and a mutant-specific primer that does not amplify the WT allele (Table 1). The amounts of mRNA of the NLaz ${ }^{\text {L130R }}$ mutant version are similar to those of the WT transcript in the transgenic flies (Supplementary Figure 1d). The expression levels of NLaz transcripts in flies expressing NLaz ${ }^{\mathrm{N} 165 \mathrm{~S}}$ or NLaz ${ }^{\Delta 177-192}$ are similar to those of WT over-expresser (Supplementary Figure 1d).

\section{Fly handling, lifespan, stress sensitivity and behavioral assays.}

Flies were grown in standard laboratory conditions as described (19). Fly survival was assayed in standard media for lifespan determination ( $\mathrm{n}=76-147$ male flies/ genotype), in vials with filter papers soaked in $10 \%$ sucrose-20 $\mathrm{mM}$ paraquat (PQ) (Sigma), for oxidative stress sensitivity measurement, or in empty vials for starvation-desiccation sensitivity measurements $(n=67-153$ male flies/genotype/condition) as previously described (18). Tubes containing the flies were genotype-blinded, and the counting of dead flies in the numbered tubes was randomly performed by two trained observers (M.R. and a lab technician). All tests were carried out at $25^{\circ} \mathrm{C}$.

To drive the expression of the NLaz transgenes, the driver da:Gal4 (ubiquitous expression) line was crossed with pUASt-NLaz fly lines or $w^{1118}$ flies (to generate driver-only control flies).

The absence of infection by Wolbachia pipiens in our fly strains (Supplementary Figure 1c was tested as described (18).

Courtship and mating tests were performed by a trained observer (M.R.) as described (18). The courtship index, representing the proportion of time a male spends actively courting the female for the first $10 \mathrm{~min}$, was scored in 25-30 couples/genotype. 
Wild-type flies (expressing the driver alone, $\mathrm{w}^{1118}$; da:Gal4/+) were used as control in over-expression experiments, whereas NLaz-KO flies ( $\mathrm{w}^{1118}$; NLaz ${ }^{\mathrm{NW} 5} / \mathrm{NLaz}^{\mathrm{NW} 5}$; da:Gal4/+) are the control in rescue experiments. All UAS:NLaz transgenes are located in the same locus of the genome $(86 \mathrm{Fb}$ in chromosome 3$)$.

\section{Metabolic Measurements and AGE assay.}

Flies (4-5 day-old) were studied in basal situation and after 8 hours of starvation. Two independent groups of 15 flies per genotype and condition were analyzed. A set of kits were used for triglycerides (BioSystems, Spain), glucose (Sigma, USA) and glycogen (Sigma, USA) measurements, following the manufacturers' instructions. Absorbance was measured by a Versamax microplate reader (Molecular Devices).

Fluorescent AGEs (advanced glycation end products) were measured as described (22).

\section{Quantitative RT-PCR gene expression array.}

RNA from pools of 25 homogenized flies per genotype and condition was extracted and reverse transcribed as previously described (18). We used SYBR Green and designed a custom qRT-PCR array (SABiosciences) with 4 replicas per sample to study gene expression changes generated by the expression of $\mathrm{NLaz}^{\mathrm{WT}}$ or its mutant versions. RNA transcription profiles were determined by the method of direct comparison of $\mathrm{Ct}$ values and relative quantities calculated by the $\Delta \Delta \mathrm{Ct}$ method (50). The list of genes explored and their relationship to the physiological output that motivated each gene selection is shown in the Supplementary Data File 1. A fold regulation $\geq 2$ and $p<0.05$ for at least one genotype was established for the gene to be considered as a PQ or aging-responsive gene (Supplementary data file 2).

\section{Statistical analyses.}

Statistical analyses were performed with SigmaPlot (v 11.0) software. A $p<0.05$ was defined as a threshold for significant changes. ANOVA was used when assaying for multiple comparisons. The particular tests used for post-hoc analyses depended on homoscedasticity, and are stated in figure legends. Multiple survival curves were compared with a log-rank test followed by Holm-Sidak multiple comparison post-hoc test.

\section{RESULTS}

In order to design mutations that could result in altered ligand binding properties we modeled the NLaz tertiary structure in the Swiss Model platform (Figure 1a-b). The average RMSD of the NLaz model is $3.71 \AA$, and that for the $\beta$-sheet and $\alpha$-helices is $0.74 \AA$. The QMEAN quality estimate is 0.707 , passing the threshold for a reliable model. Based on the well known ligand-protein interactions of the closely related ApoD (31) we designed a binding pocket mutant (NLaz ${ }^{\mathrm{L} 130 \mathrm{R}}$; Figure $\left.1 \mathrm{~b}\right)$ predicted to hinder ligand binding by introducing a charged residue with a large side chain facing the pocket. Different mutations were then selected as controls. An NLaz allele with a serine in position 165 was found in our wild-type fly stocks, while an asparagine in that position appears in the NLaz sequence of the genome Drosophila database. In this work, the N165 version was selected as the reference wild-type protein, matching the current entries in genomic databases. The $\mathrm{NLaz}^{\mathrm{N} 165 \mathrm{~S}}$ version might represent a polymorphism present in laboratory flies. Since N165 lies outside the binding pocket (Figure 1a and Supplementary Figure 2a), it has the potential to show the 
physiological outcomes of expressing an NLaz point mutant unrelated to ligand binding. We also explored the effect of deleting a C-terminal extension (residues 177192, Figure 1a and Supplementary Figure 2d,e), which is unique to NLaz within the lipocalin family (51), as a different control for the NLaz ${ }^{\mathrm{L} 130 \mathrm{R}}$ binding pocket mutant. This C-terminal tail is similar in size to the GPI-anchoring signal peptide present in the lipocalin Lazarillo of grasshoppers and other insects $(22,52)$, but shows a quite different amino acid composition.

\section{Monitoring NLaz-ligand interactions in vitro by intrinsic fluorescence analysis}

The proteins of interest were expressed using an inducible expression system in Drosophila S2 cells. The proteins were purified from the culture medium by affinity chromatography against a C-terminal His-tag followed by two rounds of size exclusion chromatography to reach a purity $>99 \%$ (Supplementary Figure 1a). The proteins produced by $\mathrm{S} 2$ cells were successfully secreted into the culture medium, are glycosylated and show signs of intramolecular disulfide bonds, as expected for a correctly folded native NLaz protein (Supplementary Figure 1b). We centered our biochemical analysis on the ligand binding properties of $\mathrm{NLaz}^{\mathrm{WT}}$ and $\mathrm{NLaz}^{\mathrm{L} 130 \mathrm{R}}$.

We used tryptophan fluorescence titration to assay the interaction of NLaz with different ligands (Figure 1c-h). One of the two tryptophan residues in NLaz, W128, is predicted to be located within the binding pocket. Its modification by interaction with ligands in the pocket could either quench or enhance its fluorescence emission. Alltrans-retinoic acid (RA) produced quenching of the emitted fluorescence (Figures $1 \mathrm{c}, \mathrm{f})$ in both, $\mathrm{NLaz}^{\mathrm{WT}}$ and $\mathrm{NLaz}^{\mathrm{L} 130 \mathrm{R}}$. RA binding, which is present in most lipocalins tested so far (29, 53-55), substantiates the correct folding of the S2 expressed proteins. We then tested ergosterol, the functional equivalent to mammalian cholesterol in insects, and proved that it produces an enhanced fluorescence over the carrier baseline in NLaz ${ }^{\mathrm{WT}}$ (Figure 1d), but not in $\mathrm{NLaz}^{\mathrm{L} 130 \mathrm{R}}$ (Figure 1g). The same pattern was observed with the pheromone 7(z)-tricosene (56) (Figures 1e,h) demonstrating that the alteration of the pocket introduced by the L130R mutation was compromising the binding to ligands of different sizes, molecular properties and presumed biological functions. Similar apparent $K d$ for the ligand-protein interaction have been obtained for RA-lipocalins by us and others $(28,29)$. Ergosterol and $7(\mathrm{z})$ tricosene have been tested for the wild type version of NLaz showing a $\mathrm{Kd}$ of 2.7 $\mu \mathrm{M}$ and $4.5 \mu \mathrm{M}$ respectively (23). However, no binding was detected in the micromolar range for $\mathrm{NLaz}^{\mathrm{L} 130 \mathrm{R}}$.

\section{$\mathrm{NLaz}^{\mathrm{WT}}$ has stronger cell survival promoting activity than $\mathrm{NLaz}^{\mathrm{L130R}}$ under oxidative stress in a cell culture-based assay.}

In order to test the biological activity of the wild type and ligand binding-altered NLaz, we treated non-transfected $\mathrm{S} 2$ cell cultures with $\mathrm{H}_{2} \mathrm{O}_{2}$ and measured cell viability by trypan blue exclusion analysis (Figure 2a). Neither version of NLaz protein nor an unrelated control protein (Trypsin Inhibitor) had effects on the viability of untreated cells. Upon a pro-oxidant treatment the viability of control cells was reduced to $45 \%$. No rescue was observed when the culture was supplemented with the control protein, but a significant increase in viability was obtained with both NLaz ${ }^{\mathrm{WT}}$ and $\mathrm{NLaz}^{\mathrm{L} 130 \mathrm{R}}$. The increase in viability produced by $\mathrm{NLaz}^{\mathrm{WT}}$ was nevertheless significantly higher than that obtained with the pocket mutant $\mathrm{NLaz}^{\mathrm{L} 130 \mathrm{R}}$. 


\section{Monitoring the survival promoting activity of $\mathrm{NLaz}^{\mathrm{WT}}$ and mutant forms in vivo.}

The wild type and mutant versions of NLaz were placed downstream of an UAS (upstream activated sequence)-containing promoter to control their expression by the Gal4/UAS system in Drosophila. Using PhiC31-mediated transformation all the transgenes were integrated into the fly genome in position $86 \mathrm{Fb}$ of chromosome 3 , an otherwise neutral site with no phenotypic effects known due to insertion mutations (See Methods section). This strategy aims at ameliorating differences of genetic background and positional effect in expression levels (see Supplementary Figure 1d) and tissue distribution, thus allowing a proper comparison of the effects of different NLaz versions on the fly physiological parameters. In this work we study the ubiquitous expression of the transgenes using the daughterless:Gal4 (da:Gal4) driver. The combination of this driver with the single-site responding transgenes allows for comparisons across genotypes throughout the life of the fly.

\section{Drosophila resistance to oxidative stress throughout aging is differentially modulated by NLaz mutant versions.}

We previously demonstrated that the expressions of NLaz and its mammalian homologue ApoD are naturally induced, via JNK signaling activation, upon exposure to the oxidative stress generator paraquat $(\mathrm{PQ})(14,16)$. The expression of NLaz/ApoD is required to promote survival of both flies and mice under oxidative stress conditions $(5,16,57)$.

When flies are exposed to PQ and $\mathrm{NLaz}^{\mathrm{WT}}$ is overexpressed with a ubiquitous pattern (da:Gal4 driver), young 3 day-old flies increase moderately their survival (Figure 2b), but aged flies (30 day-old) experience a 13.6 and $40.2 \%$ increase in median and maximal survival respectively (Figure $2 \mathrm{c}$ and Table 2 ). In contrast, overexpression of the pocket mutant $\mathrm{NLaz}^{\mathrm{L} 130 \mathrm{R}}$ does not increase, but causes a reduction in the survival of flies (Figures 2b-c and Table 2) with a 13.5 and $8.9 \%$ decrease in median and maximal survival of WT aged flies respectively. When the expression of $\mathrm{NLaz}^{\mathrm{WT}}$ or $\mathrm{NLaz}^{\mathrm{L} 130 \mathrm{R}}$ is performed in the NLaz null background (flies homozygous for the allele $\mathrm{NLaz}^{\mathrm{NW} 5}$ ) the ligand binding-altered version cannot rescue the sensitivity to PQ to the level attained by expressing the wild type NLaz version (Figure 2d). Taken together, these results are in agreement with a need of a native ligand binding activity for NLaz to exert its protective effect upon oxidative stress.

$\mathrm{NLaz}^{\mathrm{N} 165 \mathrm{~S}}$, bearing a mutated residue located outside the binding pocket (Supplementary Figure 2a), results in extended survival upon PQ treatment in young flies in wild type background, as well as in flies lacking native NLaz (Supplementary Figures 2b-c, and Table 2). Whether this NLaz allele represents a stress resistance promoting polymorphism existing in naturally occurring fly populations deserves further studies.

The mutant NLaz ${ }^{\Delta 177-192}$ is also predicted not to alter the ligand binding properties of NLaz. It results in a survival curve similar to $\mathrm{NLaz}^{\mathrm{WT}}$ in young flies exposed to PQ (Supplementary Figure 2d). In the NLaz null mutant background this truncated version of NLaz produces a partial rescue of median survival, but a complete rescue of maximal survival (Supplementary Figure 2e and Table 2). These data again reinforce the idea that the altered ligand binding pocket generated by the L130R mutation, unlike other NLaz protein modifications, decreases significantly the NLaz protective effects upon PQ exposure. 
A native binding pocket is required to rescue the Drosophila longevity reduction phenotype of the NLaz null mutation.

Since resistance to oxidative stress is often correlated with extended longevity, we tested the ability of $\mathrm{NLaz}^{\mathrm{L} 130 \mathrm{R}}$ to rescue the decrease in lifespan caused by the absence of NLaz (16).

Expressing the ligand binding-altered $\mathrm{NLaz}^{\mathrm{L} 130 \mathrm{R}}$ in the NLaz null background results in a lifespan shorter than that of the null mutant (Figure 2e, Table 2). This result is compatible with a requirement for a native ligand binding activity in NLaz in order to obtain a wild type-like longevity pattern. However, it also suggests a potential dominant-negative effect of the $\mathrm{NLaz}^{\mathrm{L} 130 \mathrm{R}}$ protein. Interactions of $\mathrm{NLaz}^{\mathrm{L} 130 \mathrm{R}}$ with NLaz functional partners might be more deleterious than the absence of NLaz.

Aging-related markers accumulate early in NLaz null mutant, and their rescue is dependent on NLaz pocket integrity.

We have previously shown that the accumulation of oxidized forms of sugars (AGEs), which are reliable biomarkers of normal aging-related damage, is accelerated in the NLaz null mutants (22). The increase in fluorescent AGEs caused by the lack of NLaz is fully rescued by the ubiquitous expression of NLaz ${ }^{\mathrm{WT}}$, but not by $\mathrm{NLaz}^{\mathrm{L} 130 \mathrm{R}}$ (Figure $3 a)$, indicating that the beneficial action of NLaz in the delay of age-related damage is dependent on its ligand binding ability.

Fly reproductive behavior also depends on NLaz binding pocket structure.

Since parameters controlling reproduction have also an impact on the rate of aging and longevity, we explored male courtship performance, which is significantly reduced in null NLaz flies ((18) and Figure 3b). NLaz ${ }^{\text {WT }}$ rescues this phenotype, while the courtship index of males expressing NLaz ${ }^{\text {L130R }}$ is more similar to the null mutant flies than to the wild type control (Figure 3b). This result, together with the inability of NLaz ${ }^{\mathrm{L} 130 \mathrm{R}}$ to bind a key male pheromone (Figure 1h), suggests that NLaz binding to specific ligands contributes to the mechanisms controlling courtship behavior.

\section{Drosophila resistance to metabolic stress is affected by NLaz ligand binding pocket structure.}

Resistance to metabolic stress is also correlated with an extended longevity, and we have described how NLaz expression can be triggered upon starvation-desiccation conditions and modulates metabolism by acting on the IIS pathway (16).

Consequently, a loss of NLaz decreases resistance of flies to the lack of nutrients and water. As expected, the over-expression of $\mathrm{NLaz}^{\mathrm{WT}}$ significantly increases the resistance of NLaz null flies to starvation-desiccation stress (Figure 2f, Table 2). However, the NLaz ${ }^{\mathrm{L} 130 \mathrm{R}}$ point mutant exhibits only a partial rescue of the null mutant phenotype (Figure 2f, Table 2).

Measurements of metabolic state: NLaz binding pocket integrity does not affect the metabolic response to starvation. 
Energy management and metabolism is tightly linked to longevity regulation and the rate of aging (reviewed by 12). We have previously shown that triglyceride, glucose and glycogen levels are decreased during starvation levels in NLaz null mutants than in NLaz WT (16). NLaz is induced in response to stress (16) or to a high sugar diet (21), and exerts a negative regulation on the IIS pathway.

Here we explore the effects of eight hours of starvation-desiccation stress on triglycerides, glucose and glycogen levels of flies expressing NLaz ${ }^{\text {WT }}$ and NLaz ${ }^{\text {L130R }}$ in the null mutant background. The depletion of triglycerides, glucose and glycogen is significantly larger in the absence of NLaz (Figure 4a-c). This phenotype is either partially or totally rescued by both $\mathrm{NLaz}^{\mathrm{WT}}$ and $\mathrm{NLaz}^{\mathrm{L} 130 \mathrm{R}}$. Therefore, energy storage management in response to starvation appears to be unaffected by the altered binding pocket of NLaz ${ }^{\mathrm{L} 130 \mathrm{R}}$.

\section{Transcriptional responses to oxidative stress or aging are modulated by NLaz and are differentially rescued by $\mathrm{NLaz}^{\mathrm{WT}}$ and $\mathrm{NLaz}^{\mathrm{L} 130 \mathrm{R}}$ transgenes.}

The expression of a set of 40 genes was explored in the same four fly genotypes as above (Figure 5) using a custom qRT-PCR array (see Methods). Genes were selected to monitor the state of pathways regulating metabolism, aging and stress biomarkers, lipid and carbohydrate metabolism, and pheromonal and neuropeptide signaling. The other two known Drosophila lipocalin genes (GLaz and Karl) were also included together with genes related to the innate immune response, given the infectionprotective effect known for Karl (16). In addition to technical controls, four potential housekeeping genes were included. Among those, in agreement with the criteria of Ponton et al. (58), we found that RpL32 is the one with least variation among genotypes and conditions, and was used as a reference for the analysis. Each genotype was explored under basal conditions (3 day-old flies), upon PQ exposure (17 h treatment, 3 day-old flies), and with aging (30 day-old flies).

In untreated young flies, the differences between genotypes are small, and only three genes (Dpt, Karl and llp4) fulfill our criteria of altered expression (see Methods) in the NLaz null mutant with respect to heterozygous control flies (Table S2 in Supplementary data file 1). Among these, the expression pattern of the lipocalin Karl is of particular interest: its expression decreases in the NLaz null mutant and is recovered by both $\mathrm{NLaz}^{\mathrm{WT}}$ and $\mathrm{NLaz}^{\mathrm{L} 130 \mathrm{R}}$ ubiquitous expression.

PQ exposure provoked a transcriptional response in 23 of the 40 genes studied while aging modified the expression of 24 genes (Supplementary data file 2). In Figure 5 we show the subsets of responding genes where $\mathrm{NLaz}^{\mathrm{WT}}$ or $\mathrm{NLaz}^{\mathrm{L} 130 \mathrm{R}}$ revert the transcriptional change observed in the NLaz null mutant. NLaz ubiquitous expression is able to rescue the effects of PQ in 12 genes (Figure 5a), representing over half the sample of responding genes (Figure 5b). Interestingly, NLaz overexpression rescues the expression of 17 aging-dependent genes (Figure 5c), representing three quarters of the sample (Figure 5b). Furthermore, a differential rescue by the $\mathrm{NLaz}^{\mathrm{WT}}$ transgene is particularly enriched in the aging-dependent gene sample with approximately half the rescuing effects being dependent on a native NLaz ligand binding pocket (binding pocket dependent vs. independent fractions in Figure 5b).

In the transcriptional response to PQ we found three genes whose expression is differentially rescued by the $\mathrm{NLaz}^{\mathrm{WT}}$ transgene (boxed in Figure 5a). They are functionally related to lifespan modulation (Lsp2, (59)), detoxification, oxidative and heat stress response (GstE1, (60)), and regulation of neurogenesis by metabolism 
(Ilp4, (61)). In the response to aging more genes show binding pocket-dependent rescue by NLaz (boxed in Figure 5c). They include genes directly involved in the IIS pathway activity (InR, (12)) and oxidative stress responsive genes that are in turn regulated by the IIS pathway (Gadd45, (62)), genes downstream of the stressresponsive JNK pathway (puc, Karl and Hsp68, $(16,63)$ ), genes related to the immune response (the antibacterial peptide Drs and Karl, $(16,64)$ ), aging-modulated neuropeptides and synaptic proteins (Nplp3 and synaptogyrin (59)), as well as neuropeptides controlling food-intake (sNPF, also downstream of IIS pathway (6567)).

Among the tested genes, NLaz binding pocket-dependent transcriptional changes were not observed for genes that we had selected for their direct relationship to pheromone synthesis, lipid homeostasis or lipid metabolism.

\section{DISCUSSION}

In this study we have tested in vivo the requirement of a native ligand binding activity for the lipocalin NLaz to fulfill its physiological functions.

The mutation L130R renders NLaz unable to bind different types of ligands (ergosterol and 7(z)-tricosene). This modification hinders the survival promoting activity of NLaz, both in a cell-based culture system and in vivo, where it is unable to rescue the effects of the NLaz null mutation upon oxidative or metabolic stress. $\mathrm{NLaz}^{\mathrm{L} 130 \mathrm{R}}$ does not rescue the accumulation of an age-dependent marker, and it appears to show a dominant-negative effect in lifespan modulation. Therefore, the residue L130 appears to be a key element in the ligand binding abilities of NLaz in vivo, as it has been shown in vitro for its human homologue ApoD. The crystal structure of hApoD bound to progesterone, a ligand of the same structural class as ergosterol, shows the residue L129 (equivalent to L130 in NLaz) in direct contact with the ligand (31). When modeling the binding of a very different lipid, arachidonic acid, the same residue shows up as an interacting partner in hApoD (31). L130 lies in close proximity to a tryptophan residue conserved in the entire lipocalin family (W127 in hApoD, W128 in NLaz), which is also known to be involved in the proteinligand interaction and is probably responsible for most of the intrinsic fluorescence changes observed upon ligand binding in our assays. However, the L130R substitution is predicted to alter a particular sub-site of the binding pocket. Therefore, as confirmed by our results, the binding of chemically distinct ligands can be differentially affected.

Our analysis also shows that the ligand binding-altered version of NLaz $\left(\mathrm{NLaz}^{\mathrm{L} 130 \mathrm{R}}\right.$ ) cannot rescue courtship behavioral phenotypes. Our data suggest a specific function of NLaz as a mediator of pheromone signals, either at modulating their availability in the extracellular milieu or its reception by the sensory neurons in charge.

Also, the transcriptional changes monitored in our study clearly show that NLaz acts upstream of key pathways modulating the management of resources upon stress or aging. Many of the genes with NLaz-dependent responses to aging or PQ are in fact genes regulated by the IIS pathway, either as direct FOXO targets (Lip4, Gadd45, Sod2, Pepck, and SNPF) or downstream of the TOR pathway (Thor). This further supports our previous findings of a negative control of IIS activity by NLaz (16), and suggests additional consequences derived from NLaz function. Noticeably, only some 
of these transcriptional rescues are dependent on the binding pocket modification of $\mathrm{NLaz}^{\mathrm{L} 130 \mathrm{R}}$.

Our results show that altering the binding pocket environment differentially affects a subset of NLaz functions. Aging and stress resistance regulation are part of an ancestral set of functions conserved in NLaz-related homologues $(5,57,68,69)$. Since these roles are affected overall by a single site mutation in the pocket that prevents binding of some potential ligands, the integrity of the inter-molecular interactions involving the conserved NLaz L130 residue is clearly required for these functions. Another important finding of our study is that a native binding pocket is not required to rescue some NLaz-dependent metabolic phenotypes and a significant proportion of transcriptional phenotypes downstream of NLaz action upon PQ or aging. These data challenge the view that the integrity of the ligand binding pocket is essential for all molecular mechanisms of action of NLaz.

The new findings help us to refine our previous conclusions about modulation of lifespan through the control of metabolism under stress conditions by NLaz (16). We previously proposed that the lifespan expanding activity of NLaz was due to the systemic action of fat body-derived NLaz on peripheral target tissues, inhibiting IIS and therefore repressing growth (16). Also, we showed that a constitutive lack of NLaz function significantly increases the hunger-driven food intake behavior of the flies, and that they gain weight (particularly fat) with aging (18). Now we find that NLaz mutants have an aging-dependent increased expression of SNPF, a gene involved in food intake and body size (66). This result provides a possible explanation for the food intake and "age-related obesity" phenotypes, and highlights new actions of NLaz within the CNS. NLaz might exert a negative regulation of the expression of SNPF downstream of FOXO in the neuropeptidergic neurons, thus contributing, in addition to its systemic control, to the final metabolic-nutritional status and lifespan of the flies.

We also find that under oxidative stress NLaz modulates the expression of Ilp4 in a binding pocket-dependent manner. Ilp4 is one of the insulin-like peptides described to be part of the CNS specific pool, and it is known to contribute to reactivate neurogenesis during larval development (61), but not to organismal growth. Curiously, NLaz does not alter the expression of Ilp2, Ilp3 or Ilp5 (16), the systemic pool of insulin-like peptides in Drosophila that control organismal growth.

In summary, our work, using NLaz as a model lipocalin and taking advantage of standardized Drosophila genetic tools, opens the way to identify residues in the binding pocket of lipocalins that are required for complex physiological roles tested in a living organism. These and future results studying other residues in the lipocalin hydrophobic pocket will allow us to experimentally address in vivo the more general question of whether ligand binding can account for the full panoply of Lipocalin actions in the organism.

\section{AUTHOR'S CONTRIBUTIONS}

M.R. designed and performed the experiments, analyzed the data and helped in writing the manuscript. D.S. and M.D.G, designed the research project and helped with experiments and analysis of results, C.C. and R.K.S. participated in the 
discussion and design of experiments. M.D.G., D.S. and M.R. wrote the manuscript. All authors have read and approved the final manuscript.

\section{ACKNOWLEDGMENTS}

We thank Dr. J.M. Pereda (CIC, Salamanca, Spain) for providing advice on potential residues to mutate in NLaz. Gene sequencing was performed in the Genomic facility of the Centro de Investigación del Cancer (CIC, Salamanca, Spain). Drosophila transgenesis was carried out in BestGene Inc. (California, USA). E. Martin-Tejedor provided technical assistance throughout the project. We also want to acknowledge the critical and constructive comments of two anonymous reviewers that have certainly improved the manuscript and the accuracy of data presentation and interpretation herein included.

Grant support. This work was supported by grants to M.D.G. and D.S. (Junta de Castilla y León (JCyL) grant VA180A11-2, and Ministerio de Ciencia e Innovación (MICINN) grants BFU2008-01170 and BFU2011-23978), and M.R. was supported by a JCyL fellowship to young researchers (call\#EDU/1708/2008).

\section{REFERENCES}

1. Akerström, B., Borregaard, N., Flover, D., and Salier, J. (2006) Lipocalins, Georgetown, Texas

2. Kawaguchi, R., Yu, J., Honda, J., Hu, J., Whitelegge, J., Ping, P., Wiita, P., Bok, D., and Sun, H. (2007) A membrane receptor for retinol binding protein mediates cellular uptake of vitamin A. Science 315, 820-825

3. Correnti, C., and Strong, R. K. (2012) Mammalian siderophores, siderophore-binding lipocalins, and the labile iron pool. J Biol Chem 287, 13524-13531

4. Wade, N. M., Anderson, M., Sellars, M. J., Tume, R. K., Preston, N. P., and Glencross, B. D. (2012) Mechanisms of colour adaptation in the prawn Penaeus monodon. The Journal of Experimental Biology 215, 343-350

5. Ganfornina, M. D., Do Carmo, S., Lora, J. M., Torres-Schumann, S., Vogel, M., Allhorn, M., Gonzalez, C., Bastiani, M. J., Rassart, E., and Sanchez, D. (2008) Apolipoprotein D is involved in the mechanisms regulating protection from oxidative stress. Aging Cell 7, 506-515

6. Berger, T., Togawa, A., Duncan, G. S., Elia, A. J., You-Ten, A., Wakeham, A., Fong, H. E., Cheung, C. C., and Mak, T. W. (2006) Lipocalin 2-deficient mice exhibit increased sensitivity to Escherichia coli infection but not to ischemia-reperfusion injury. Proc Natl Acad Sci U S A 103, 1834-1839

7. Eguchi, N., Minami, T., Shirafuji, N., Kanaoka, Y., Tanaka, T., Nagata, A., Yoshida, N., Urade, Y., Ito, S., and Hayaishi, O. (1999) Lack of tactile pain (allodynia) in lipocalin-type prostaglandin D synthase-deficient mice. Proc Natl Acad Sci U S A 96, 726-730

8. Vogel, S., Piantedosi, R., O'Byrne, S. M., Kako, Y., Quadro, L., Gottesman, M. E., Goldberg, I. J., and Blaner, W. S. (2002) Retinol-binding protein-deficient mice: biochemical basis for impaired vision. Biochemistry 41, 15360-15368

9. Sanchez, D., Ganfornina, M. D., Gutierrez, G., and Marin, A. (2003) Exon-intron structure and evolution of the Lipocalin gene family. Mol Biol Evol 20, 775-783 
10. Gutierrez, G., Ganfornina, M. D., and Sanchez, D. (2000) Evolution of the lipocalin family as inferred from a protein sequence phylogeny. Biochim Biophys Acta 1482, 35-45

11. Ganfornina, M. D., Gutierrez, G., Bastiani, M., and Sanchez, D. (2000) A phylogenetic analysis of the lipocalin protein family. Mol Biol Evol 17, 114-126

12. Kourtis, N., and Tavernarakis, N. (2011) Cellular stress response pathways and ageing: intricate molecular relationships. EMBO J 30, 2520-2531

13. Sanchez, D., Ganfornina, M. D., Torres-Schumann, S., Speese, S. D., Lora, J. M., and Bastiani, M. J. (2000) Characterization of two novel lipocalins expressed in the Drosophila embryonic nervous system. Int J Dev Biol 44, 349-359

14. Bajo-Graneras, R., Ganfornina, M. D., Martin-Tejedor, E., and Sanchez, D. (2011) Apolipoprotein D mediates autocrine protection of astrocytes and controls their reactivity level, contributing to the functional maintenance of paraquat-challenged dopaminergic systems. Glia 59, 1551-1566

15. Bajo-Graneras, R., Sanchez, D., Gutierrez, G., Gonzalez, C., Do Carmo, S., Rassart, E., and Ganfornina, M. D. (2011) Apolipoprotein D alters the early transcriptional response to oxidative stress in the adult cerebellum. J Neurochem 117, 949-960

16. Hull-Thompson, J., Muffat, J., Sanchez, D., Walker, D. W., Benzer, S., Ganfornina, M. D., and Jasper, H. (2009) Control of metabolic homeostasis by stress signaling is mediated by the lipocalin NLaz. PLoS Genet 5, e1000460

17. Navarro, J. A., Ohmann, E., Sanchez, D., Botella, J. A., Liebisch, G., Molto, M. D., Ganfornina, M. D., Schmitz, G., and Schneuwly, S. (2010) Altered lipid metabolism in a Drosophila model of Friedreich's ataxia. Hum Mol Genet 19, 2828-2840

18. Ruiz, M., Sanchez, D., Canal, I., Acebes, A., and Ganfornina, M. D. (2011) Sex-dependent modulation of longevity by two Drosophila homologues of human Apolipoprotein D, GLaz and NLaz. Exp Gerontol 46, 579-589

19. Sanchez, D., Lopez-Arias, B., Torroja, L., Canal, I., Wang, X., Bastiani, M. J., and Ganfornina, M. D. (2006) Loss of glial lazarillo, a homolog of apolipoprotein D, reduces lifespan and stress resistance in Drosophila. Curr Biol 16, 680-686

20. Ganfornina, M. D., Do Carmo, S., Martinez, E., Tolivia, J., Navarro, A., Rassart, E., and Sanchez, D. (2010) ApoD, a glia-derived apolipoprotein, is required for peripheral nerve functional integrity and a timely response to injury. Glia 58, 1320-1334

21. Pasco, M. Y., and Leopold, P. (2012) High sugar-induced insulin resistance in Drosophila relies on the lipocalin Neural Lazarillo. PLoS One 7, e36583

22. Ruiz, M., Wicker-Thomas, C., Sanchez, D., and Ganfornina, M. D. (2012) Grasshopper Lazarillo, a GPI-anchored Lipocalin, increases Drosophila longevity and stress resistance, and functionally replaces its secreted homolog NLaz. Insect Biochem Mol Biol 42, 776-789

23. Ruiz, M., Sanchez, D., Correnti, C., Strong, R. K., and Ganfornina, M. D. (2013) Lipidbinding properties of human ApoD and Lazarillo-related lipocalins: functional implications for cell differentiation. FEBS J 280, 3928-3943

24. Bhatia, S., Knoch, B., Wong, J., Kim, W. S., Else, P. L., Oakley, A. J., and Garner, B. (2012) Selective reduction of hydroperoxyeicosatetraenoic acids to their hydroxy derivatives by apolipoprotein D: implications for lipid antioxidant activity and Alzheimer's disease. Biochem $J$ 442, 713-721

25. Zhang, Y. R., Zhao, Y. Q., and Huang, J. F. (2012) Retinoid-binding proteins: similar protein architectures bind similar ligands via completely different ways. PLoS One 7, e36772 
26. Gasymov, O. K., Abduragimov, A. R., and Glasgow, B. J. (2010) pH-Dependent conformational changes in tear lipocalin by site-directed tryptophan fluorescence. Biochemistry 49, 582-590

27. Tcatchoff, L., Nespoulous, C., Pernollet, J. C., and Briand, L. (2006) A single lysyl residue defines the binding specificity of a human odorant-binding protein for aldehydes. FEBS Lett 580, 2102-2108

28. Sanchez, D., Ortega-Cubero, S., Akerstrom, B., Herrera, M., Bastiani, M. J., and Ganfornina, M. D. (2008) Molecular interactions of the neuronal GPI-anchored lipocalin Lazarillo. J Mol Recognit 21, 313-323

29. Breustedt, D. A., SchÃ $\llbracket$ nfeld, D. L., and Skerra, A. (2006) Comparative ligand-binding analysis of ten human lipocalins. Biochimica et Biophysica Acta (BBA) - Proteins and Proteomics 1764, 161-173

30. Vogt, M., and Skerra, A. (2001) Bacterially produced apolipoprotein D binds progesterone and arachidonic acid, but not bilirubin or E-3M2H. Journal of Molecular Recognition 14, 7986

31. Eichinger, A., Nasreen, A., Kim, H. J., and Skerra, A. (2007) Structural Insight into the Dual Ligand Specificity and Mode of High Density Lipoprotein Association of Apolipoprotein D. Journal of Biological Chemistry 282, 31068-31075

32. Morais Cabral, J. H., Atkins, G. L., Sanchez, L. M., Lopez-Boado, Y. S., Lopez-Otin, C., and Sawyer, L. (1995) Arachidonic acid binds to apolipoprotein D: implications for the protein's function. FEBS Lett 366, 53-56

33. Elenewski, J. E., and Hackett, J. C. (2010) Free energy landscape of the retinol/serum retinol binding protein complex: a biological host-guest system. J Phys Chem B 114, 11315-11322

34. Kalikka, J., and Akola, J. (2011) Steered molecular dynamics simulations of ligand-receptor interaction in lipocalins. Eur Biophys J 40, 181-194

35. Chiswell, B., Lovelace, L. L., Brannen, C., Ortlund, E. A., Lebioda, L., and Sodetz, J. M. (2007) Structural features of the ligand binding site on human complement protein C8gamma: a member of the lipocalin family. Biochim Biophys Acta 1774, 637-644

36. Gasymov, O. K., Abduragimov, A. R., and Glasgow, B. J. (2009) Intracavitary ligand distribution in tear lipocalin by site-directed tryptophan fluorescence. Biochemistry 48, 72197228

37. Zhou, Y., Shaw, N., Li, Y., Zhao, Y., Zhang, R., and Liu, Z. J. (2010) Structure-function analysis of human 1-prostaglandin D synthase bound with fatty acid molecules. FASEB J 24, 4668-4677

38. Abergel, R. J., Clifton, M. C., Pizarro, J. C., Warner, J. A., Shuh, D. K., Strong, R. K., and Raymond, K. N. (2008) The siderocalin/enterobactin interaction: a link between mammalian immunity and bacterial iron transport. J Am Chem Soc 130, 11524-11534

39. Correnti, C., Clifton, M. C., Abergel, R. J., Allred, B., Hoette, T. M., Ruiz, M., Cancedda, R., Raymond, K. N., Descalzi, F., and Strong, R. K. (2011) Galline Ex-FABP is an antibacterial siderocalin and a lysophosphatidic acid sensor functioning through dual ligand specificities. Structure 19, 1796-1806

40. Kumasaka, T., Aritake, K., Ago, H., Irikura, D., Tsurumura, T., Yamamoto, M., Miyano, M., Urade, Y., and Hayaishi, O. (2009) Structural basis of the catalytic mechanism operating in open-closed conformers of lipocalin type prostaglandin D synthase. J Biol Chem 284, 2234422352 
41. Sundaram, M., Sivaprasadarao, A., Aalten, D. M., and Findlay, J. B. (1998) Expression, characterization and engineered specificity of rat epididymal retinoic acid-binding protein. Biochem J 334 ( Pt 1), 155-160

42. Biesalski, H. K., Frank, J., Beck, S. C., Heinrich, F., Illek, B., Reifen, R., Gollnick, H., Seeliger, M. W., Wissinger, B., and Zrenner, E. (1999) Biochemical but not clinical vitamin A deficiency results from mutations in the gene for retinol binding protein. Am J Clin Nutr 69, 931-936

43. Gebauer, M., and Skerra, A. (2012) Anticalins small engineered binding proteins based on the lipocalin scaffold. In Methods Enzymol Vol. 503 pp. 157-188

44. Fukuhara, A., Yamada, M., Fujimori, K., Miyamoto, Y., Kusumoto, T., Nakajima, H., and Inui, T. (2012) Lipocalin-type prostaglandin D synthase protects against oxidative stressinduced neuronal cell death. Biochem J 443, 75-84

45. Kiefer, F., Arnold, K., Kunzli, M., Bordoli, L., and Schwede, T. (2009) The SWISS-MODEL Repository and associated resources. Nucleic Acids Res 37, D387-392

46. Kopp, J., and Schwede, T. (2004) The SWISS-MODEL Repository of annotated threedimensional protein structure homology models. Nucleic Acids Res 32, D230-234

47. Benkert, P., Schwede, T., and Tosatto, S. C. (2009) QMEANclust: estimation of protein model quality by combining a composite scoring function with structural density information. BMC Struct Biol 9, 35

48. Rong, Y. S., Titen, S. W., Xie, H. B., Golic, M. M., Bastiani, M., Bandyopadhyay, P., Olivera, B. M., Brodsky, M., Rubin, G. M., and Golic, K. G. (2002) Targeted mutagenesis by homologous recombination in D. melanogaster. Genes Dev 16, 1568-1581

49. Bischof, J., Maeda, R. K., Hediger, M., Karch, F., and Basler, K. (2007) An optimized transgenesis system for Drosophila using germ-line-specific phiC31 integrases. Proc Natl Acad Sci U S A 104, 3312-3317

50. Livak, K. J., and Schmittgen, T. D. (2001) Analysis of relative gene expression data using real-time quantitative PCR and the 2(-Delta Delta C(T)) Method. Methods 25, 402-408

51. Ganfornina, M. D., Kayser, H., and Sanchez, D. (2006) Lipocalins in Arthropoda: Diversification and functional explorations. In Lipocalins (Åkerström, B., Borregaard, N., Flower, D. R., and Salier, J.-P., eds) pp. 49-74, Landes Bioscience, Georgetown, Texas

52. Ganfornina, M. D., Sanchez, D., and Bastiani, M. J. (1995) Lazarillo, a new GPI-linked surface lipocalin, is restricted to a subset of neurons in the grasshopper embryo. Development 121, $123-134$

53. Ahnstrom, J., Faber, K., Axler, O., and Dahlback, B. (2007) Hydrophobic ligand binding properties of the human lipocalin apolipoprotein M. J Lipid Res 48, 1754-1762

54. Peng, Y., Liu, J., Liu, Q., Yao, Y., Guo, C., Zhang, Y., and Lin, D. (2010) Conformational and biochemical characterization of a rat epididymis-specific lipocalin 12 expressed in Escherichia coli. Biochim Biophys Acta 1804, 2102-2110

55. Zsila, F., Bikadi, Z., and Simonyi, M. (2002) Retinoic acid binding properties of the lipocalin member beta-lactoglobulin studied by circular dichroism, electronic absorption spectroscopy and molecular modeling methods. Biochem Pharmacol 64, 1651-1660

56. Grillet, M., Dartevelle, L., and Ferveur, J. F. (2006) A Drosophila male pheromone affects female sexual receptivity. Proc Biol Sci 273, 315-323 
57. Muffat, J., Walker, D. W., and Benzer, S. (2008) Human ApoD, an apolipoprotein upregulated in neurodegenerative diseases, extends lifespan and increases stress resistance in Drosophila. Proc Natl Acad Sci U S A 105, 7088-7093

58. Ponton, F., Chapuis, M. P., Pernice, M., Sword, G. A., and Simpson, S. J. (2011) Evaluation of potential reference genes for reverse transcription-qPCR studies of physiological responses in Drosophila melanogaster. J Insect Physiol 57, 840-850

59. Bauer, J., Antosh, M., Chang, C., Schorl, C., Kolli, S., Neretti, N., and Helfand, S. L. (2010) Comparative transcriptional profiling identifies takeout as a gene that regulates life span. Aging (Albany NY) 2, 298-310

60. Curtis, C., Landis, G. N., Folk, D., Wehr, N. B., Hoe, N., Waskar, M., Abdueva, D., Skvortsov, D., Ford, D., Luu, A., Badrinath, A., Levine, R. L., Bradley, T. J., Tavare, S., and Tower, J. (2007) Transcriptional profiling of MnSOD-mediated lifespan extension in Drosophila reveals a species-general network of aging and metabolic genes. Genome Biol 8, R262

61. Sousa-Nunes, R., Yee, L. L., and Gould, A. P. (2011) Fat cells reactivate quiescent neuroblasts via TOR and glial insulin relays in Drosophila. Nature 471, 508-512

62. Moskalev, A. A., Smit-McBride, Z., Shaposhnikov, M. V., Plyusnina, E. N., Zhavoronkov, A., Budovsky, A., Tacutu, R., and Fraifeld, V. E. (2012) Gadd45 proteins: relevance to aging, longevity and age-related pathologies. Ageing Res Rev 11, 51-66

63. Wang, M. C., Bohmann, D., and Jasper, H. (2003) JNK signaling confers tolerance to oxidative stress and extends lifespan in Drosophila. Dev Cell 5, 811-816

64. Wu, S. C., Liao, C. W., Pan, R. L., and Juang, J. L. (2012) Infection-induced intestinal oxidative stress triggers organ-to-organ immunological communication in Drosophila. Cell Host Microbe 11, 410-417

65. Lee, K. S., Kwon, O. Y., Lee, J. H., Kwon, K., Min, K. J., Jung, S. A., Kim, A. K., You, K. H., Tatar, M., and Yu, K. (2008) Drosophila short neuropeptide F signalling regulates growth by ERK-mediated insulin signalling. Nat Cell Biol 10, 468-475

66. Lee, K. S., You, K. H., Choo, J. K., Han, Y. M., and Yu, K. (2004) Drosophila short neuropeptide F regulates food intake and body size. J Biol Chem 279, 50781-50789

67. Kahsai, L., Kapan, N., Dircksen, H., Winther, A. M., and Nassel, D. R. Metabolic stress responses in Drosophila are modulated by brain neurosecretory cells that produce multiple neuropeptides. PLoS One 5, e11480

68. Charron, J. B., Ouellet, F., Houde, M., and Sarhan, F. (2008) The plant Apolipoprotein D ortholog protects Arabidopsis against oxidative stress. BMC Plant Biol 8, 86

69. Bishop, R. E., Penfold, S. S., Frost, L. S., Holtje, J. V., and Weiner, J. H. (1995) Stationary phase expression of a novel Escherichia coli outer membrane lipoprotein and its relationship with mammalian apolipoprotein D. Implications for the origin of lipocalins. J Biol Chem 270, 23097-23103 


\section{FIGURE AND TABLE LEGENDS}

Figure 1. NLaz primary and tertiary structures and intrinsic fluorescence analysis of NLaz-ligand interactions.

(A) $\mathrm{NLaz}^{\mathrm{WT}}$ amino acid sequence structure. Residues selected to generate point mutant versions of NLaz are boxed in gray and highlighted (L130 in red, N165 in blue). The C-terminal fragment deleted in NLaz ${ }^{\Delta 177-192}$ mutant is underlined. Some Asn residues are highlighted by rectangles; green for proved and purple for predicted glycosylation.

(B) 3D-surface model of NLaz using hApoD crystal coordinates as a template. The left panel shows wild-type NLaz with the pocket indicated by a curved arrow and the L130 residue highlighted in yellow. The right panel shows a similar view of the model for NLaz ${ }^{\mathrm{L} 130 \mathrm{R}}$ protein, with the Arg basic group marked in red and indicated by a white arrow.

(C-H) Changes in intrinsic fluorescence of $\mathrm{NLaz}^{\mathrm{WT}}$ and $\mathrm{NLaz}^{\mathrm{L} 130 \mathrm{R}}$ with different hydrophobic compounds are shown. NLaz ${ }^{\mathrm{WT}}$ fluorescence is quenched by retinoic acid (C), and enhanced by ergosterol (D) and the Drosophila pheromone 7(z)tricosene $(\mathrm{E})$. NLaz ${ }^{\mathrm{L} 130 \mathrm{R}}$ fluorescence is quenched by retinoic acid $(\mathrm{F})$, but no interaction was found for NLaz ${ }^{\mathrm{L} 130 \mathrm{R}}$ with ergosterol $(\mathrm{G})$ or $7(\mathrm{z})$-tricosene $(\mathrm{H})$.

Figure 2. NLaz ${ }^{\mathrm{L} 130 \mathrm{R}}$ shows lower survival promoting activity than $\mathrm{NLaz}^{\mathrm{WT}}$ both in cultured S2 cells and in adult flies upon aging or exposure to stress.

(A) Drosophila S2 cell viability was measured by trypan blue dye exclusion. S2 cells improve their survival upon exposure to hydrogen peroxide $\left(20 \mathrm{mM} \mathrm{H}_{2} \mathrm{O}_{2}\right)$ when 50 $\mathrm{nM}$ of exogenous pure $\mathrm{NLaz}^{\mathrm{WT}}$ or $\mathrm{NLaz}^{\mathrm{L} 130 \mathrm{R}}$ proteins are added to the culture medium. Trypsin Inhibitor (TI, $50 \mathrm{nM}$ ), added as a control unrelated protein, does not influence cell survival. Two-way ANOVA $(\mathrm{p}<0.001)$ followed by Holm-Sidak multiple comparison post-hoc test indicates that the recovery of viability obtained with NLaz ${ }^{\mathrm{WT}}$ is significantly larger than that obtained with $\mathrm{NLaz}^{\mathrm{L} 130 \mathrm{R}}$. $* *=\mathrm{p}<0.001$. (B) PQ resistance of 3 day-old flies with ubiquitous over-expression of $\mathrm{NLaz}^{\mathrm{WT}}$ or $\mathrm{NLaz}^{\mathrm{L} 130 \mathrm{R}}$ in wild type background is not significantly different from that of control flies. However, over-expression of $\mathrm{NLaz}^{\mathrm{WT}}$ confers more resistance to PQ than overexpression of $\mathrm{NLaz}^{\mathrm{L} 130 \mathrm{R}}$.

(C) Upon aging (30 day-old flies) resistance to PQ is increased by ubiquitous overexpression of $\mathrm{NLaz}^{\mathrm{WT}}$. However, old flies over-expressing $\mathrm{NLaz}^{\mathrm{L} 130 \mathrm{R}}$ are more sensitive to PQ than control flies.

(D) Three day-old flies ubiquitously over-expressing NLaz ${ }^{\mathrm{WT}}$ show a more extended survival to PQ treatment than NLaz ${ }^{\mathrm{NW} 5}$ homozygous flies. However, NLaz ${ }^{\text {L130R }}$ expression is only able to exert a partial rescue.

(E) In the absence of added stress, the reduced longevity of null NLaz fly mutants is rescued by the ubiquitous over-expression of $\mathrm{NLaz}^{\mathrm{WT}}$ (evident in terms of maximal survival; see Table 2). However, over-expression of $\mathrm{NLaz}^{\mathrm{L} 130 \mathrm{R}}$ fails to rescue NLaz loss-of-function longevity, showing a lifespan shorter than that of NLaz ${ }^{\mathrm{NW} 5}$ homozygous flies.

(F) NLaz ${ }^{\text {WT }}$ ubiquitous over-expression rescues the phenotype of sensitivity to starvation-desiccation stress in three day-old NLaz $^{\text {NW5 }}$ flies, while NLaz ${ }^{\text {L130R }}$ expression is only able to exert a partial rescue.

To compare survival distributions, log-rank tests were performed ( $p$-values shown in each panel) followed by Holm-Sidak multiple comparison post-hoc tests. Differences 
between $\mathrm{NLaz}^{\mathrm{NW} 5}$ and NLaz ${ }^{\mathrm{WT}}$ in D was further assessed by the Cox's F-test ( $p=$ $0.002)$ and the Cox-Mantel test $(p=0.004)$.

Figure 3. Aging-related markers accumulation and fly reproductive behavior are dependent on NLaz ligand-binding pocket integrity.

(A) Advanced glycation end products (AGEs), which normally accumulate upon aging, are increased at 3 and 30 days in NLaz null mutants, reflecting an accelerated aging process. NLaz ${ }^{\mathrm{WT}}$ over-expression rescues this phenotype, whereas NLaz ${ }^{\mathrm{L} 130 \mathrm{R}}$ over-expression is not able to reduce AGEs accumulation. We use heterozygous $+/ \mathrm{NLaz}^{\mathrm{NW}}$ flies with a single copy of wild type NLaz in its native locus as a control. Two-way ANOVA followed by Holm-Sidak multiple comparison post-hoc test. $* *=$ $p<0.001 ; *=p<0.005$. $\left(\mathrm{NLaz}^{\mathrm{NW} 5}, \mathrm{n}=29 ; \mathrm{NLaz}^{\mathrm{WT}}, \mathrm{n}=29 ; \mathrm{NLaz}^{\mathrm{L} 130 \mathrm{R}}, \mathrm{n}=25\right)$.

(B) Courtship behavior index is reduced in $\mathrm{NLaz}^{\mathrm{NW} 5}$ flies. NLaz ${ }^{\mathrm{WT}}$ ubiquitous overexpression rescues this phenotype completely. Although the null hypothesis cannot be rejected (the test assumes similarly shaped distributions among groups), NLaz ${ }^{\text {L130R }}$ expression results in a courtship index whose distribution is more similar in shape to the null mutant than to the wild type control. Kruskal-Wallis one-way ANOVA on ranks followed by Dunn's multiple comparison post-hoc test. $*=p<0.005$ ( $\mathrm{n}=25-30$ couples/genotype).

Figure 4. Effects of $\mathrm{NLaz}^{\mathrm{WT}}$ and $\mathrm{NLaz}^{\mathrm{L130R}}$ in the fly metabolic homeostasis. Fly metabolic measurements were performed at basal state and after $8 \mathrm{~h}$ of starvationdesiccation. Depletion of metabolite stores is represented as \% of control levels. (A) Triglyceride depletion by starvation is bigger in $\mathrm{NLaz}^{\mathrm{NW} 5}$ flies compared to control flies. Neither NLaz ${ }^{\mathrm{WT}}$ nor NLaz ${ }^{\mathrm{L} 130 \mathrm{R}}$, ubiquitously over-expressed in the $\mathrm{NLaz}^{\mathrm{NW} 5}$ genetic background, rescue the triglyceride reduction.

(B) Glucose levels after starvation also depends on NLaz expression, but alteration of the NLaz binding pocket structure does not significantly change the responses. $\mathrm{NLaz}^{\mathrm{NW5}}$ starved flies present lower levels of glucose compared to control flies, and both $\mathrm{NLaz}^{\mathrm{WT}}$ and NLaz ${ }^{\mathrm{L} 130 \mathrm{R}}$ over-expressions partially rescue this phenotype. (C) Glycogen storage depletion depends on NLaz expression, but not on NLaz binding pocket structure. Both $\mathrm{NLaz}^{\mathrm{WT}}$ and $\mathrm{NLaz}^{\mathrm{L} 130 \mathrm{R}}$ maintain equally significant higher levels of glycogen stores after starvation.

(A-B) One-way ANOVA on ranks followed by Tukey multiple comparison post-hoc tests. (C) One-way ANOVA followed by Tukey multiple comparison post-hoc tests. $* *=\mathrm{p}<0.001 ; *=\mathrm{p}<0.05$.

Figure 5. Transcriptional profile analysis shows binding pocket-dependent and independent NLaz modulation of stress and age-responsive pathways.

(A) Subset of genes where the response to PQ shows dependence on NLaz genotype. Only genes with expression changes $\geq 2(p<0.05)$ and showing a rescuing effect by either of the NLaz transgenes (fold regulation similar to the WT control) are shown. Boxes indicate genes in which the rescue is dependent on the binding pocket mutation (differential rescue). Genes with large expression changes are shown in separate graphs for scaling purposes.

(B) Percent distribution of genes with transcriptional changes upon PQ or aging classified according to the ability of NLaz transgenes to revert the changes observed in the NLaz ${ }^{\mathrm{NW} 5}$ null mutant genotype.

(C) Subset of genes whose response to aging is NLaz-dependent. Inclusion criteria as in A. Boxes indicate genes with binding pocket-dependent rescue, with dashed boxed 
showing cases with partial dependence. Genes with large expression changes are shown in separate graphs for scaling purposes.

Student's t-test was performed on the quadruplicate $2^{-\Delta \mathrm{Ct}}$ values for each gene in each genotype. Fold regulation by either PQ or aging is shown as $2^{-\Delta \Delta \mathrm{Ct}}$ when the

experimental condition produced an increase in expression, and as $-1 / 2^{-\Delta \Delta \mathrm{Ct}}$ when the condition produced down-regulation of the gene.

Table 1. Primer sequences used for PCR.

Table 2. Effects of ubiquitous expression of different versions of NLaz on fly survival and behavior. 
Figure 1

A

Signal Peptide:

MNHHSSSHLLLLISVVFGAVWVAHA

QVPFPGKCPDVKLLDTFDAEAYMGVWYEYAAYPFAFEIGK KCIYANYSLIDNSTVSVVNAAINRFTGQPSNVTGQAKVLG PGQLAVAFYPTQPLTKANYLVLGTDYESYAVVYSCTSVTP LANFKIVWILTRQREPSAEAVDAARKILEDNDVSQAFLID TVQKNCPRLDGNGTGLTGEDGLDVDDFVSTTVPNAIEKA

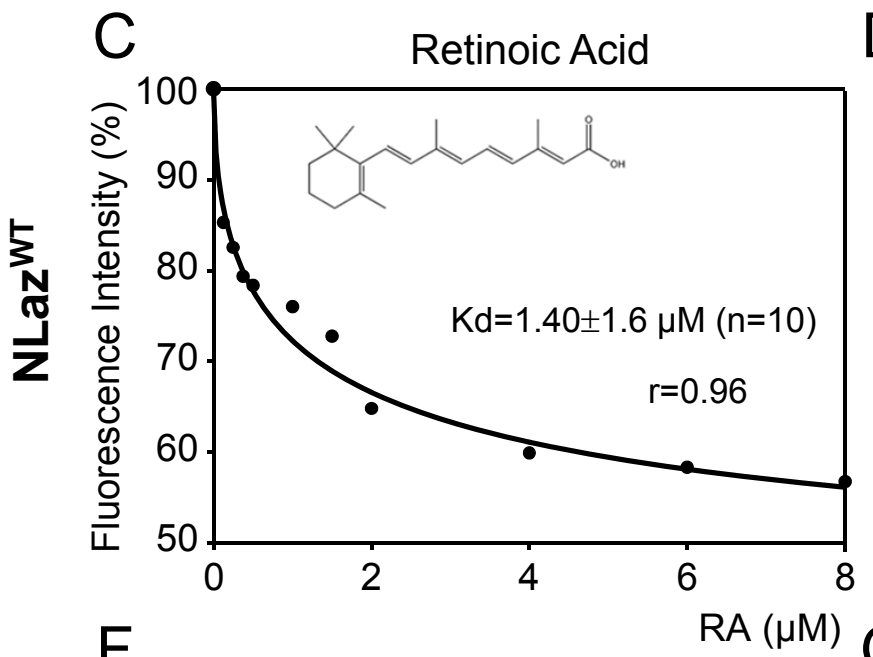

D

$\mathrm{G}$

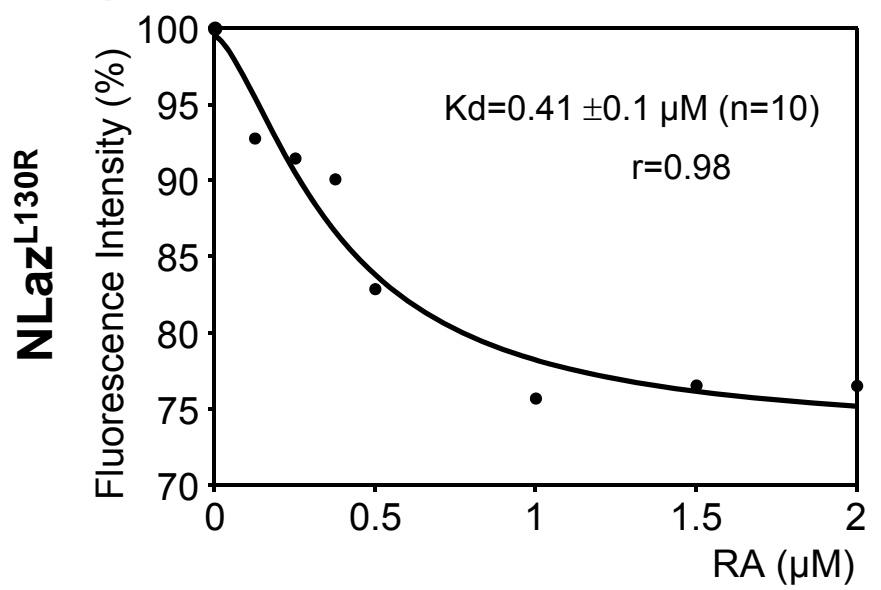

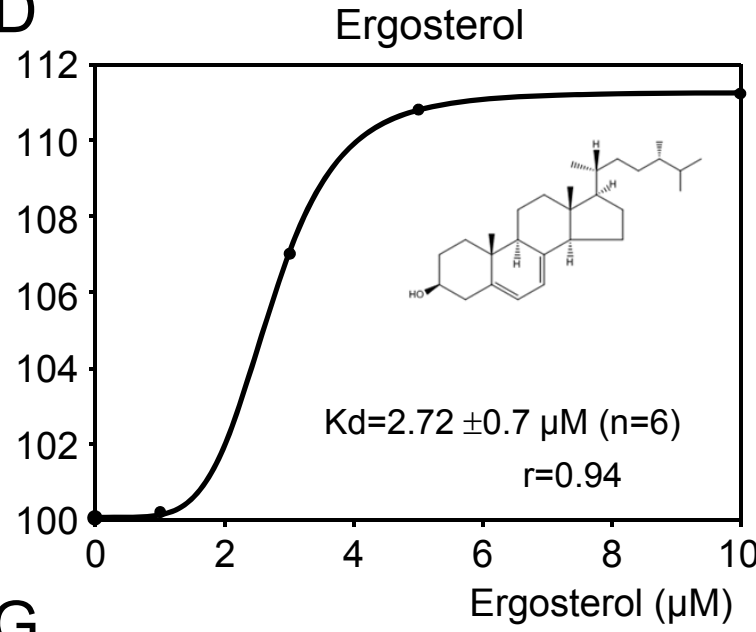

B
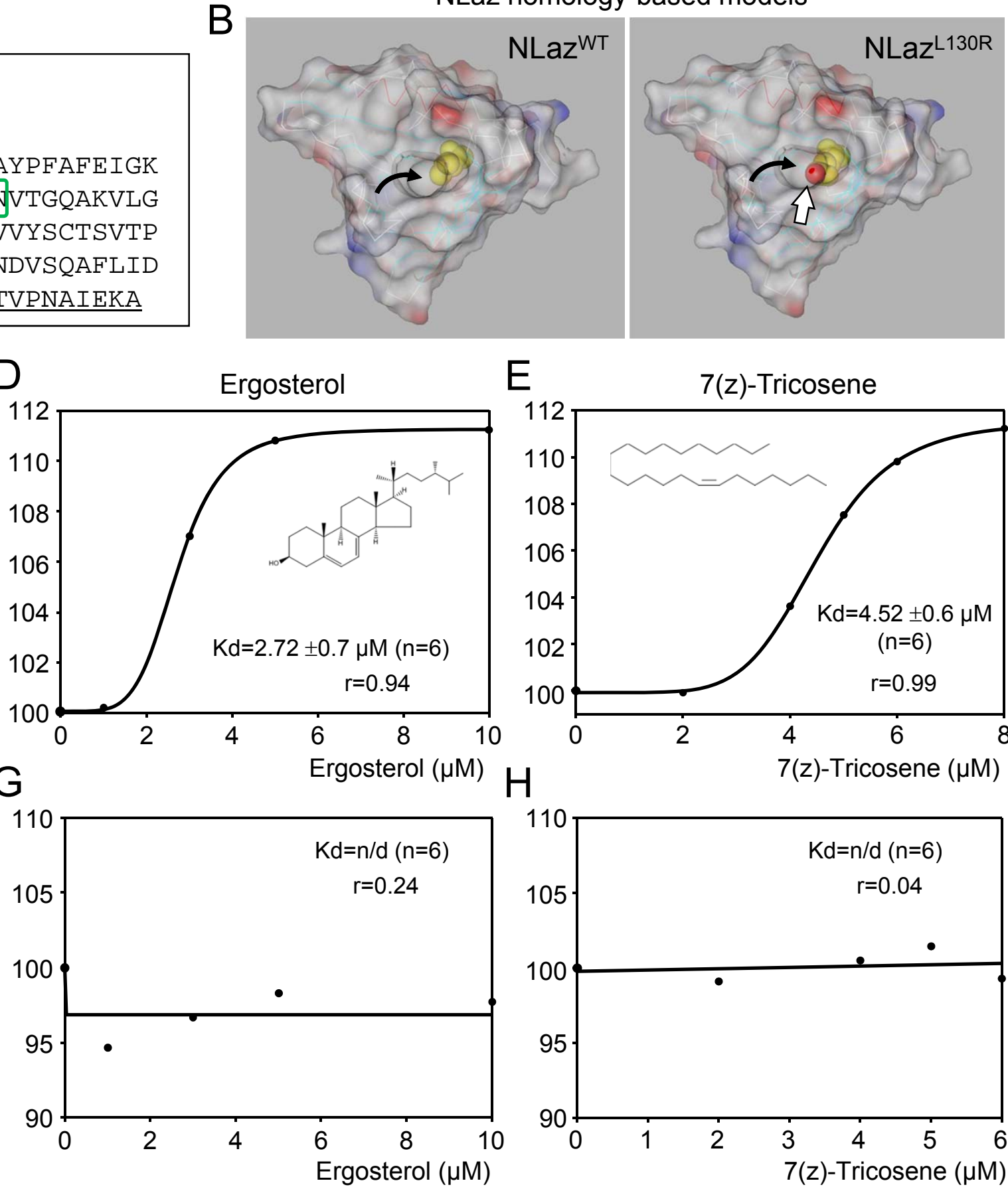

E

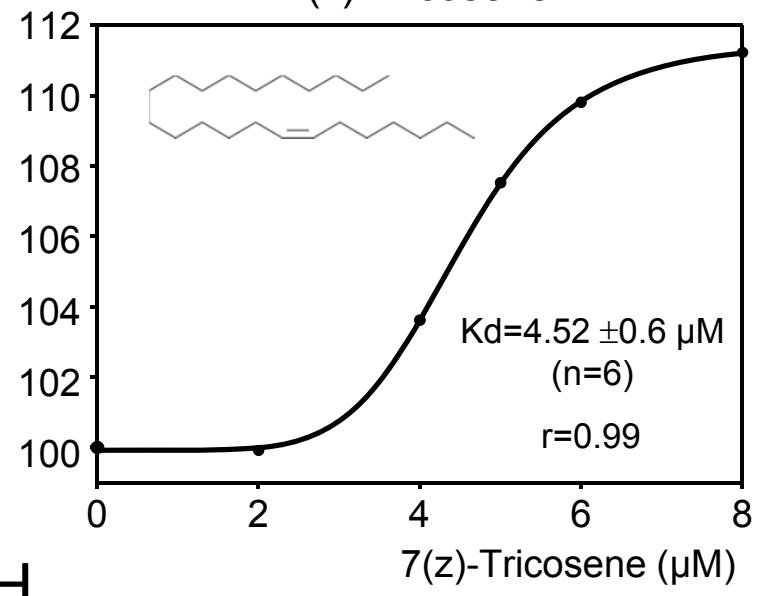

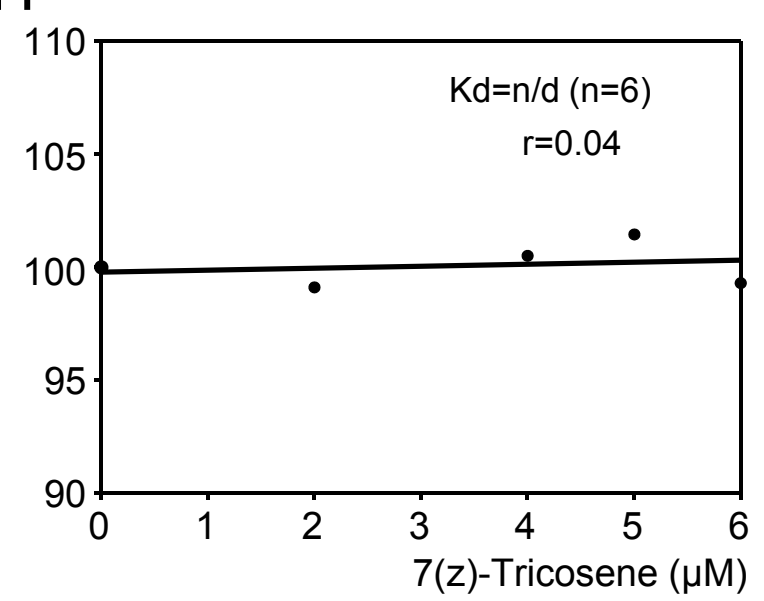




\section{Figure 2}

A Cell based assays

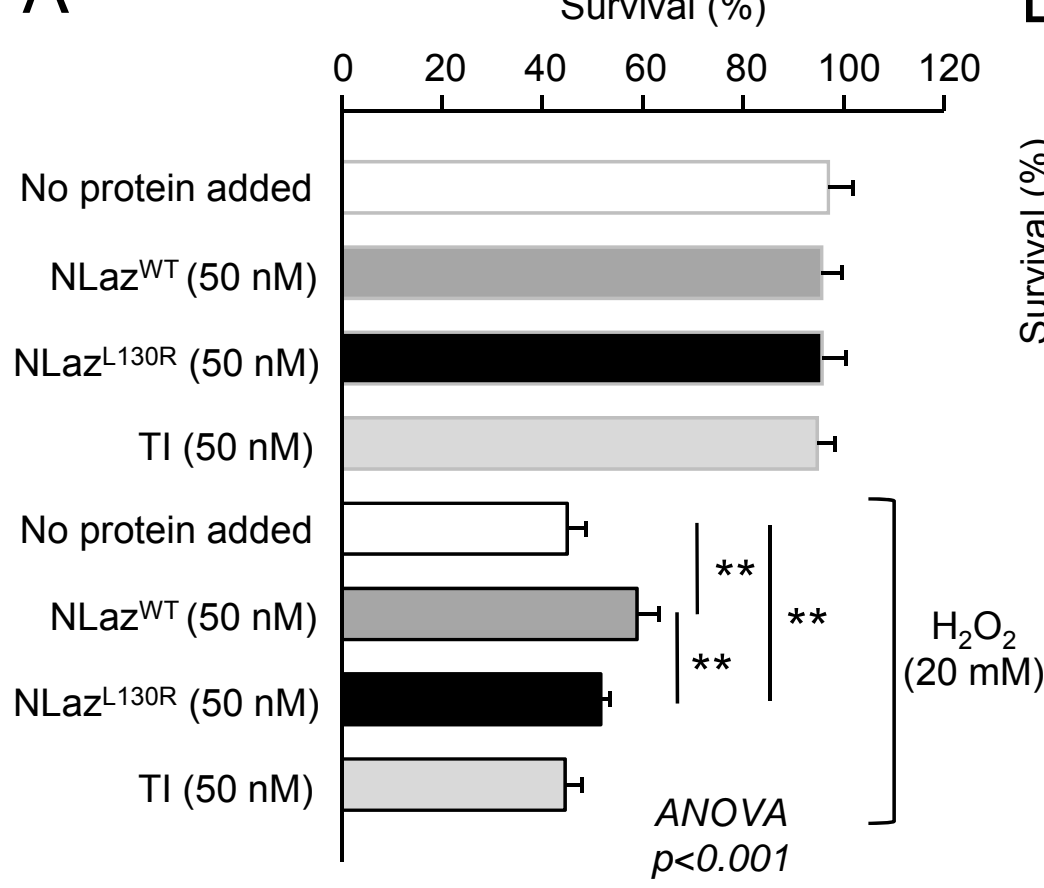

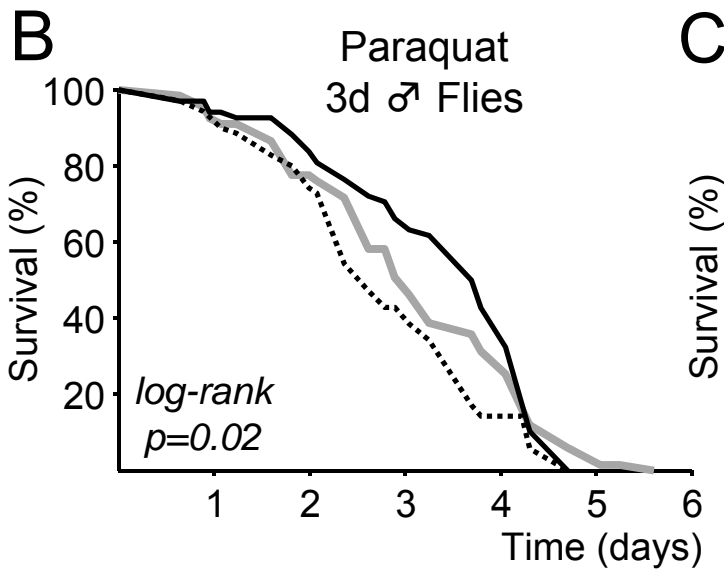
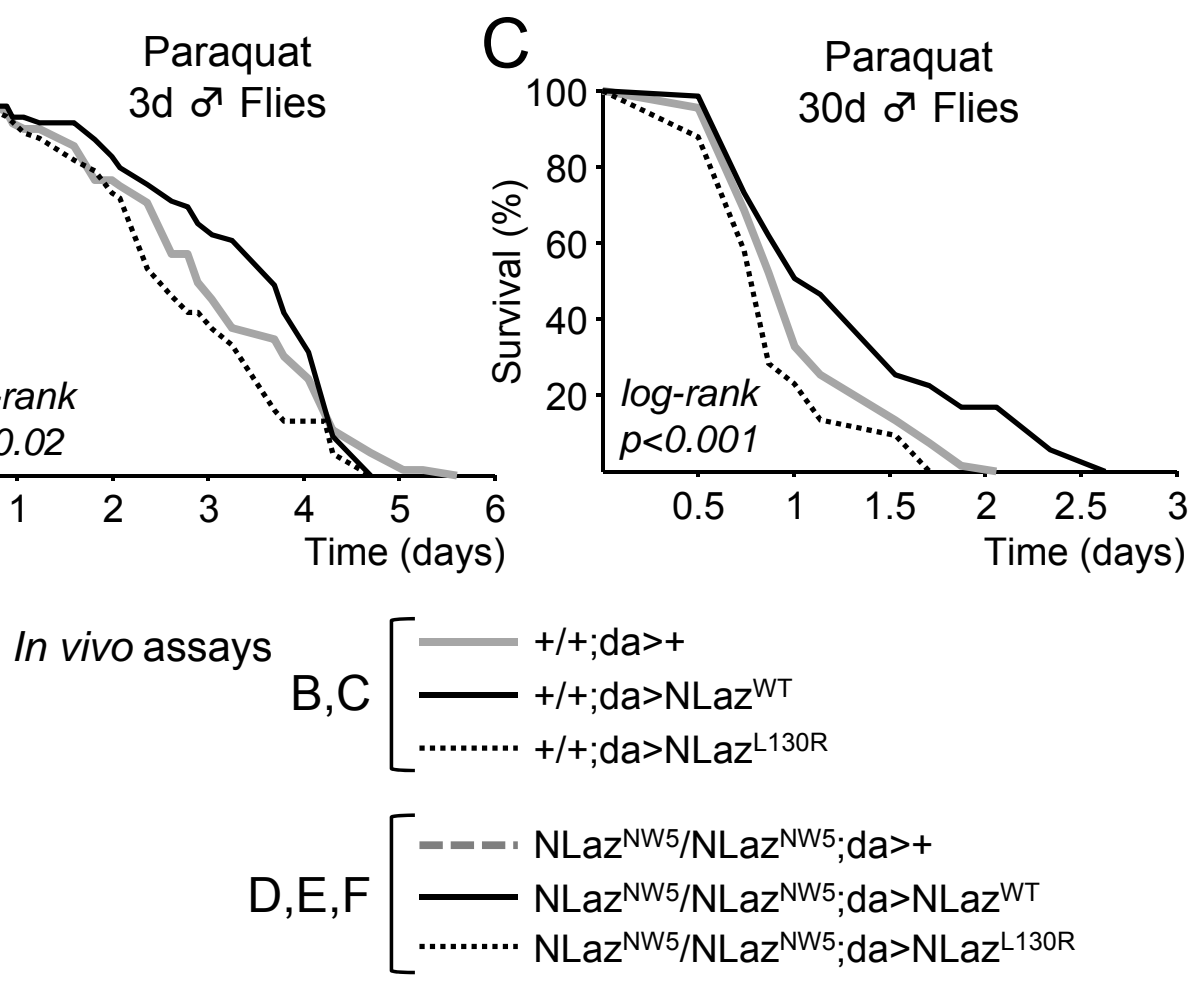
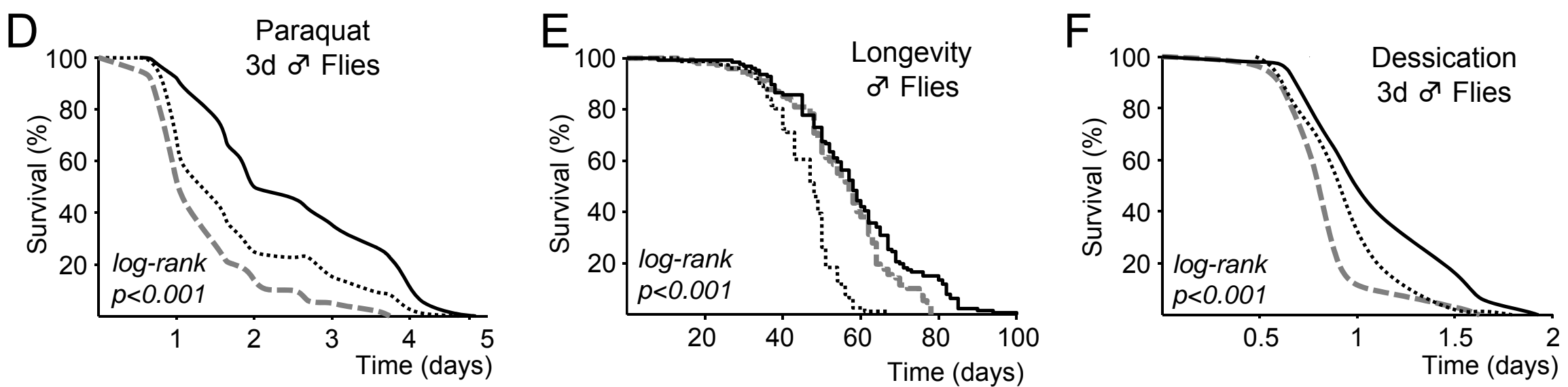


\section{Figure 3}

A Advanced glycation end-products (AGEs)

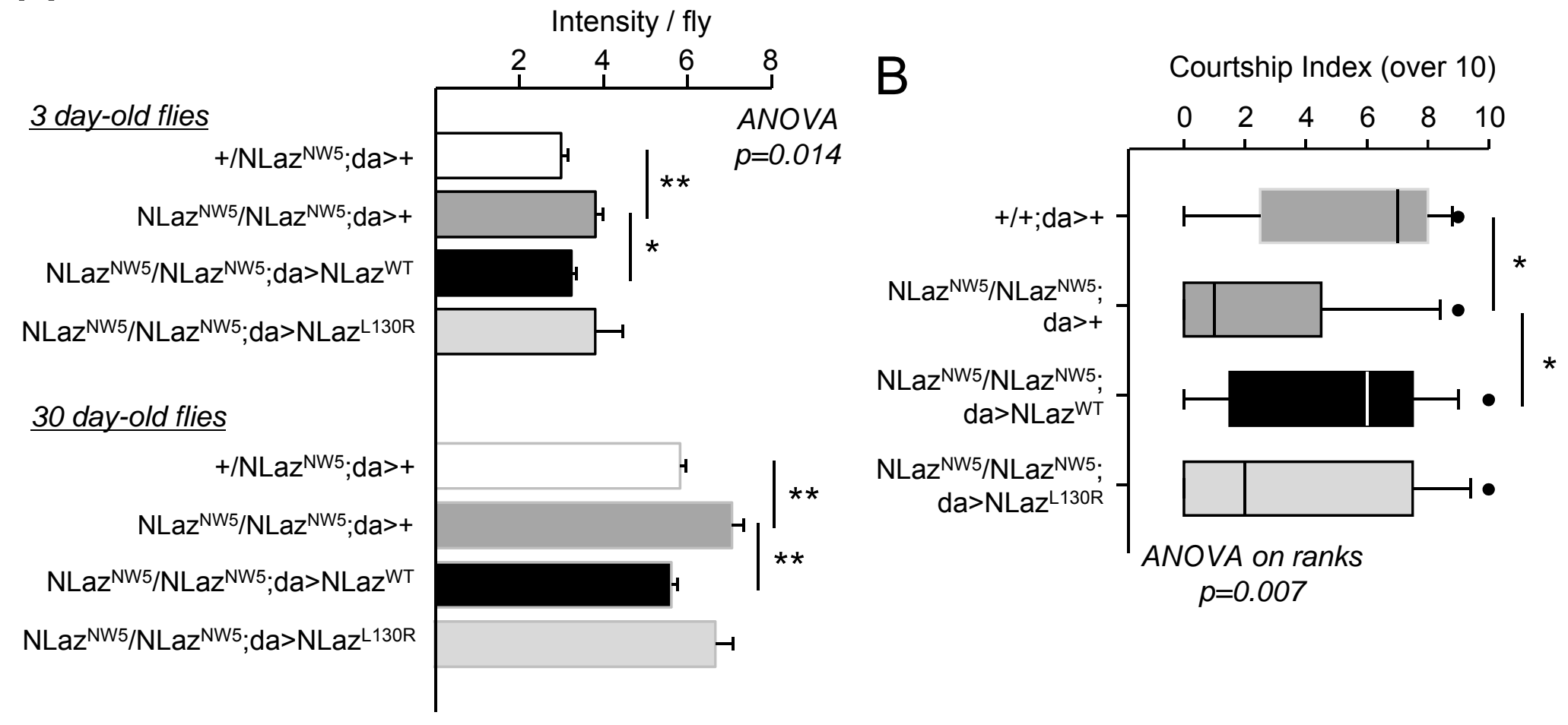


Figure 4
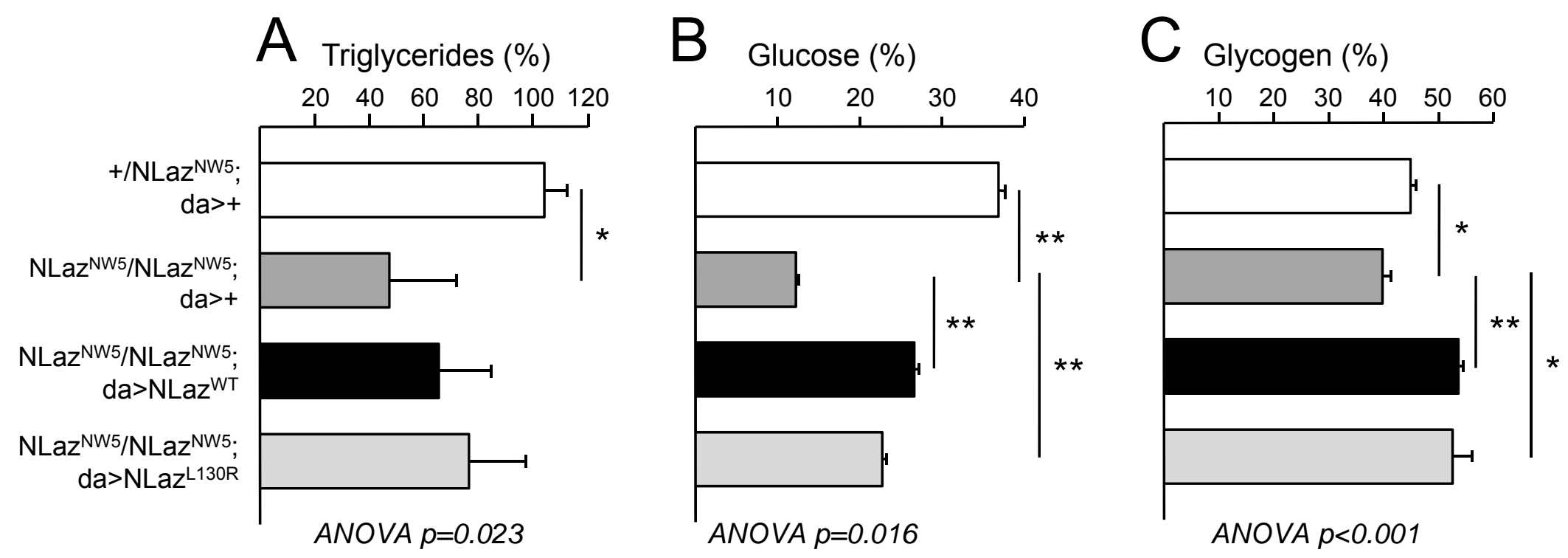


\section{Figure 5}
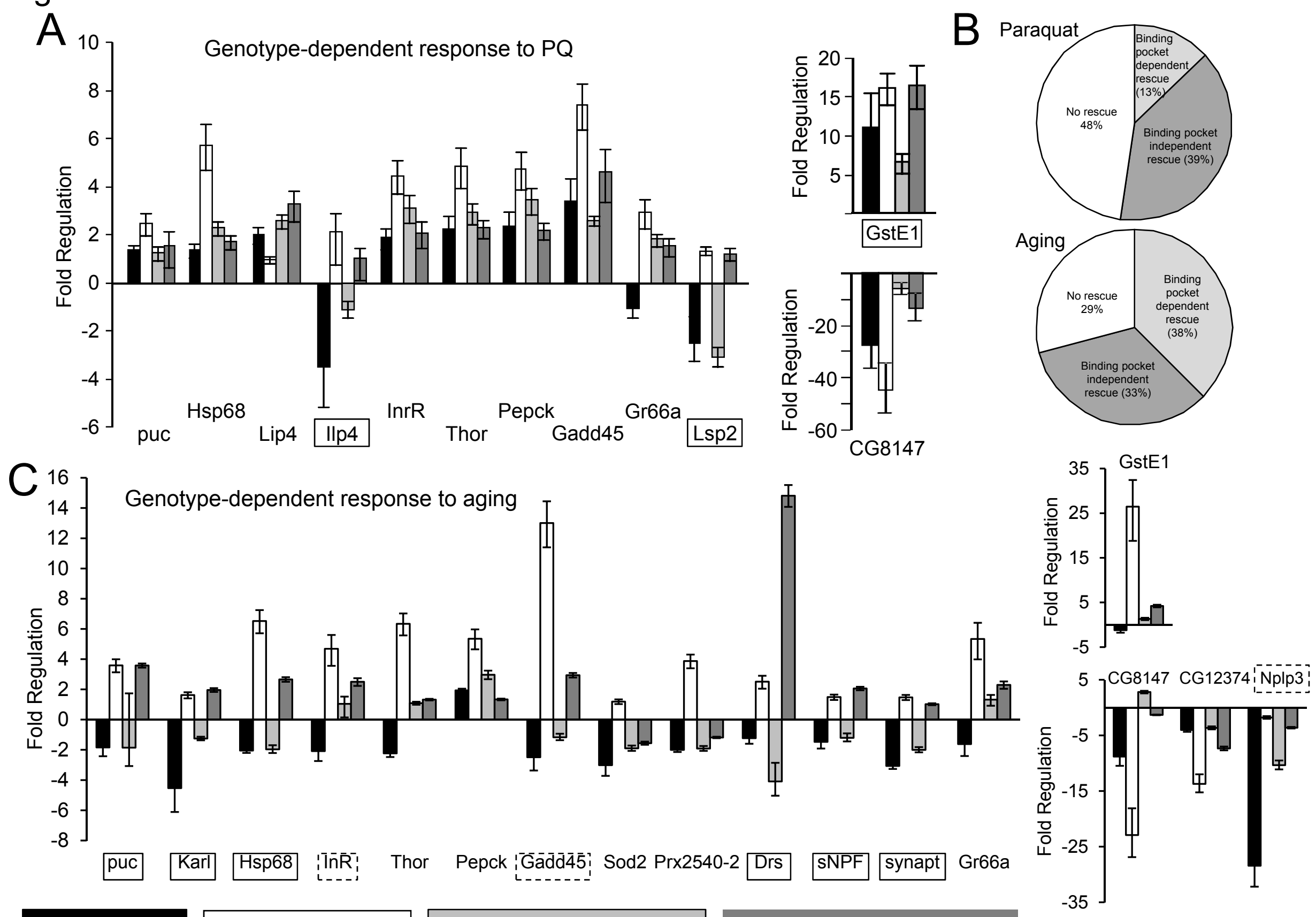
Table 1. Primer sequences.

\begin{tabular}{|c|c|}
\hline Application & Primer Sequence \\
\hline \multirow{2}{*}{ Mutagenesis L130R } & $5^{\prime}$-gccaatttcaaaattgtttggatccgcactcgtcagcgtgaaccttcagc-3' \\
\hline & $5^{\prime}$-gctgaaggttcacgctgacgagtgcggatccaaacaattttgaaattggc-3' \\
\hline \multirow{3}{*}{$\begin{array}{c}\mathrm{NLaz}^{\mathrm{WT}} \text { and } \mathrm{NLaz}^{\mathrm{L} 130 \mathrm{R}} \mathrm{qRT}- \\
\text { PCR }\end{array}$} & 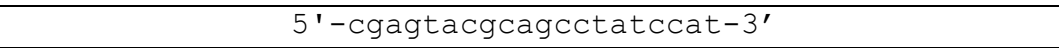 \\
\hline & $5^{\prime}-$ ccaggtagttggccttcgt-3' \\
\hline & $5^{\prime}-$ ggttcacgctgacgagtgcg-3' \\
\hline \multirow{2}{*}{ DrpL18 qRT-PCR } & 5'-agaaccgagcccaaatcc-3' \\
\hline & $5^{\prime}-\mathrm{cgaccacgatggtagactcc-3^{ \prime }}$ \\
\hline \multirow{2}{*}{ From $\mathrm{pBS}-\mathrm{II}$ to $\mathrm{pRmHa}-3$} & 5'-caagaattctcagtggatagcgac-3' \\
\hline & $5^{\prime}$-caagcggccgcggagccgcgcggcaccagtgccttttcaatggcatttgc-3' \\
\hline \multirow{2}{*}{$\begin{array}{c}\text { NLaz- } \Delta 177-192 \\
\text { from pBSII-KS to pUASt-attB }\end{array}$} & 5'-cgaattctcagtggatagcgac-3' \\
\hline & 5'-cgaattctcaaccatccaaccg-3' \\
\hline Sequence pBSII-KS & Universal Primer set (CIC) \\
\hline \multirow{2}{*}{ Sequence pUASt-attB } & 5'-gcagtaaagtgcaagttaaagtga-3' \\
\hline & 5'tgtccaattatgtcacaccac-3' \\
\hline Sequence pRmHa-3 & 5'ccagagcatctggccaatgtgc-3' \\
\hline \multirow{2}{*}{ Wolbachia rRNA-16S } & $5^{\prime}$-gaagataatgacggtactcac-3' \\
\hline & $5^{\prime \prime-g t c a g a t t t g a a c c a g a t a g a-3 '}$ \\
\hline
\end{tabular}


Table 2.Effects of ubiquitous expression of different versions of NLaz on fly survival.

\begin{tabular}{|c|c|c|c|c|c|c|c|c|}
\hline \multirow[b]{2}{*}{ Background } & \multirow[b]{2}{*}{$\begin{array}{c}\delta \mathrm{da}>\mathrm{UAS} \\
\text { transgene }\end{array}$} & \multirow[b]{2}{*}{$\mathrm{N}$} & \multirow[b]{2}{*}{ Experiment } & \multirow[b]{2}{*}{$\begin{array}{l}\text { ANOVA } \\
p \text {-value }\end{array}$} & \multicolumn{2}{|c|}{ Log Rank Test } & \multicolumn{2}{|c|}{ t-Test } \\
\hline & & & & & $p$-value & $\begin{array}{c}\text { \%Change } \\
\text { Median } \\
\text { Survival } \\
\end{array}$ & $p$-value & $\begin{array}{l}\text { \%Change } \\
\text { Maximal } \\
\text { Survival }\end{array}$ \\
\hline \multirow{4}{*}{$W T\left(w^{1118}\right)$} & $\mathrm{NLaz}^{\mathrm{N} 165 \mathrm{~S}}$ & $67 / 72$ & \multirow{4}{*}{$\begin{array}{l}\text { Paraquat } \\
\text { (3 d) }\end{array}$} & \multirow{4}{*}{0.02} & 0.005 & 38.74 & 0.318 & -6.50 \\
\hline & $\mathrm{NLaz}^{\mathrm{WT}}$ & $67 / 68$ & & & 0.533 & 23.14 & 0.073 & -6.50 \\
\hline & $\mathrm{NLaz}^{\mathrm{L} 130 \mathrm{R}}$ & $67 / 70$ & & & 0.080 & -13.92 & 0.017 & -6.50 \\
\hline & $\mathrm{NLaz}^{\Delta 177-192}$ & $67 / 71$ & & & 0.079 & 21.49 & 0.073 & -6.50 \\
\hline \multirow{2}{*}{$W T\left(w^{1118}\right)$} & $\mathrm{NLaz}^{\mathrm{WT}}$ & $67 / 71$ & \multirow{2}{*}{$\begin{array}{c}\text { Paraquat } \\
(30 \mathrm{~d})\end{array}$} & \multirow{2}{*}{$<0.001$} & 0.002 & 13.56 & $<0.001$ & 40.16 \\
\hline & $\mathrm{NLaz}^{\mathrm{L} 130 \mathrm{R}}$ & $67 / 74$ & & & 0.019 & -13.46 & 0.026 & -8.90 \\
\hline \multirow{2}{*}{$\begin{array}{c}N^{N L a z}{ }^{N W 5} \\
(\mathrm{NLaz}-\mathrm{KO})\end{array}$} & $\mathrm{NLaz}^{\mathrm{WT}}$ & $147 / 126$ & \multirow{2}{*}{ Longevity } & \multirow{2}{*}{$<0.001$} & 0.005 & 1.75 & $<0.001$ & 17.65 \\
\hline & $\mathrm{NLaz}^{\mathrm{L} 130 \mathrm{R}}$ & $147 / 76$ & & & $<0.001$ & -15.79 & $<0.001$ & -19.72 \\
\hline \multirow{4}{*}{$\begin{array}{c}N L a z^{N W 5} \\
(\mathrm{NLaz}-\mathrm{KO})\end{array}$} & $\mathrm{NLaz}^{\mathrm{N} 165 \mathrm{~S}}$ & $153 / 106$ & \multirow{4}{*}{$\begin{array}{l}\text { Paraquat } \\
(3 \mathrm{~d})\end{array}$} & \multirow{4}{*}{$<0.001$} & $<0.001$ & 181.51 & $<0.001$ & 46.04 \\
\hline & $\mathrm{NLaz}^{\mathrm{WT}}$ & $153 / 128$ & & & $<0.001$ & 90.4 & $<0.001$ & 46.04 \\
\hline & $\mathrm{NLaz}^{\mathrm{L} 130 \mathrm{R}}$ & $153 / 93$ & & & $<0.001$ & 36.09 & $<0.001$ & 23.68 \\
\hline & $\mathrm{NLaz}^{\Delta 177-192}$ & $153 / 120$ & & & $<0.001$ & 47.20 & $<0.001$ & 46.04 \\
\hline \multirow{2}{*}{$\begin{array}{c}N_{L a z}^{N W 5} \\
(\mathrm{NLaz}-\mathrm{KO})\end{array}$} & $\mathrm{NLaz}^{\mathrm{WT}}$ & $119 / 150$ & \multirow{2}{*}{ Desiccation } & \multirow{2}{*}{$<0.001$} & $<0.001$ & 22.26 & $<0.001$ & 21.74 \\
\hline & $\mathrm{NLaz}^{\mathrm{L} 130 \mathrm{R}}$ & $119 / 76$ & & & 0.002 & 22.26 & 0.964 & 0.00 \\
\hline
\end{tabular}

The first number in the third column accounts for $\mathrm{N}$ in the background sample, and the second number is $\mathrm{N}$ for the UAS transgene sample. 

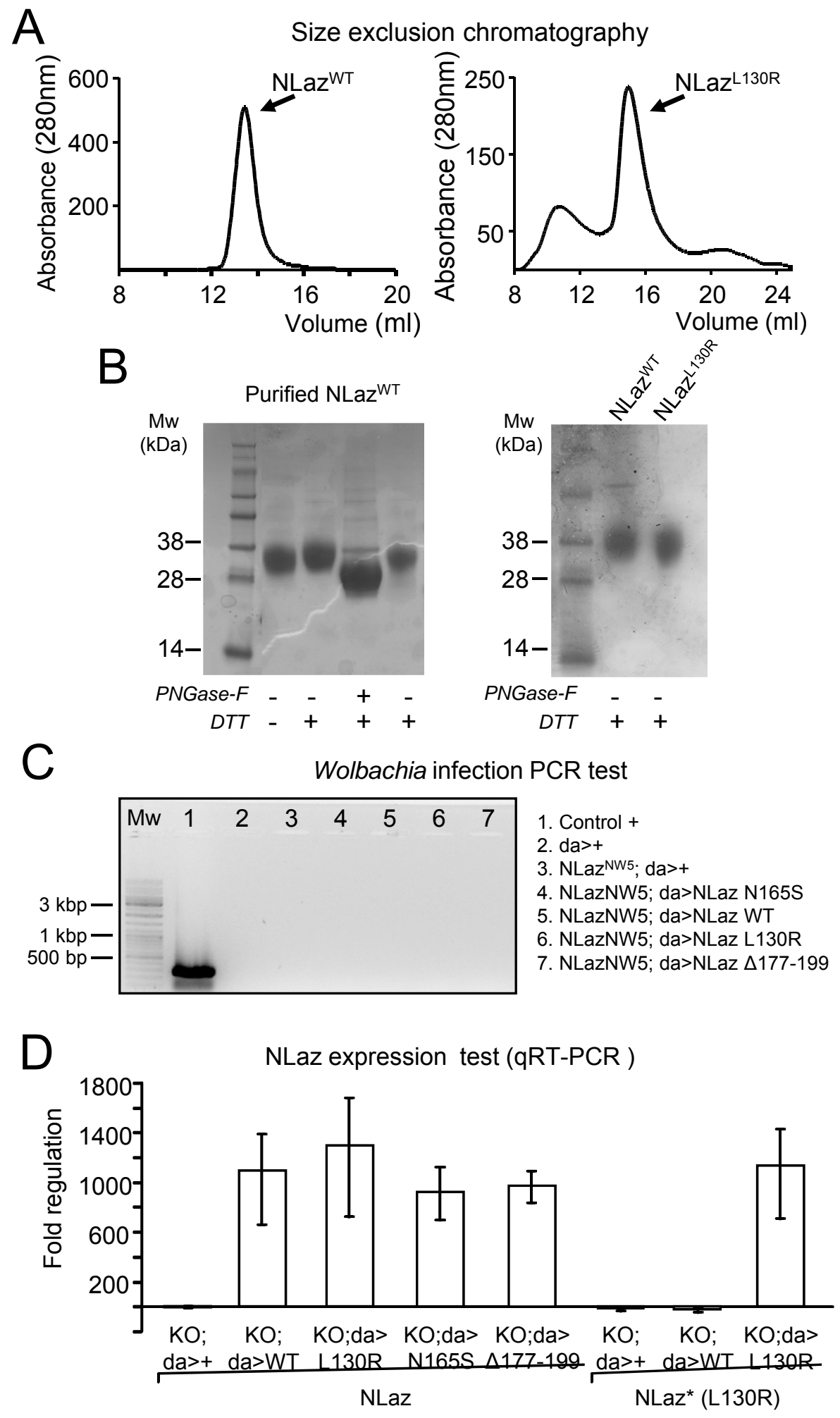

Supplementary Figure 1. Protein purifications and fly lines characterization tests.

(A) Purification of $\mathrm{NLaz}^{\mathrm{WT}}$ and $\mathrm{NLaz}^{\mathrm{L} 130 \mathrm{R}}$ from S2 cells. Size-exclusion chromatography elution profiles of enriched extracts obtained after metal affinity chromatography of culture media from stably transfected S2 cells.

(B) SDS-PAGE analysis of $\mathrm{NLaz}^{\mathrm{WT}}$ purified from $\mathrm{S} 2$ cells (left panel). The redox-state of the protein affects slightly its electrophoretic mobility, while a large shift is observed after deglycosylation. No differences in electrophoretic mobility are detected between $\mathrm{NLaz}^{\mathrm{WT}}$ and $\mathrm{NLaz}^{\mathrm{L} 130 \mathrm{R}}$ purified from $\mathrm{S} 2$ cells (right panel).

(C) Fly lines used in this study are free of Wolbachia infection. PCR of genomic DNA extracts from all lines employed. Primers against the rRNA-16S gene of Wolbachia endosymbiont were used (Table 1). DNA from Dirofilaria immitis worms infected with Wolbachia was used as a positive control.

(D) NLaz transcription levels in transgenic flies expressing $\mathrm{NLaz}^{\mathrm{WT}}, \mathrm{NLaz}^{\mathrm{L} 130 \mathrm{R}}, \mathrm{NLaz}^{\mathrm{N} 165 \mathrm{~S}}$ and $\mathrm{NLaz}^{\Delta 177-199}$. We used a pair of primers able to amplify both forms, and a mutant-specific primer that does not amplify the WT allele (Table 1). Expression NLaz levels are comparable in all over-expressors. The mRNA levels of the NLaz ${ }^{\mathrm{L} 130 \mathrm{R}}$ mutant version are similar to those of the WT transcript in the transgenic flies where we observe differences in NLaz phenotypes. 

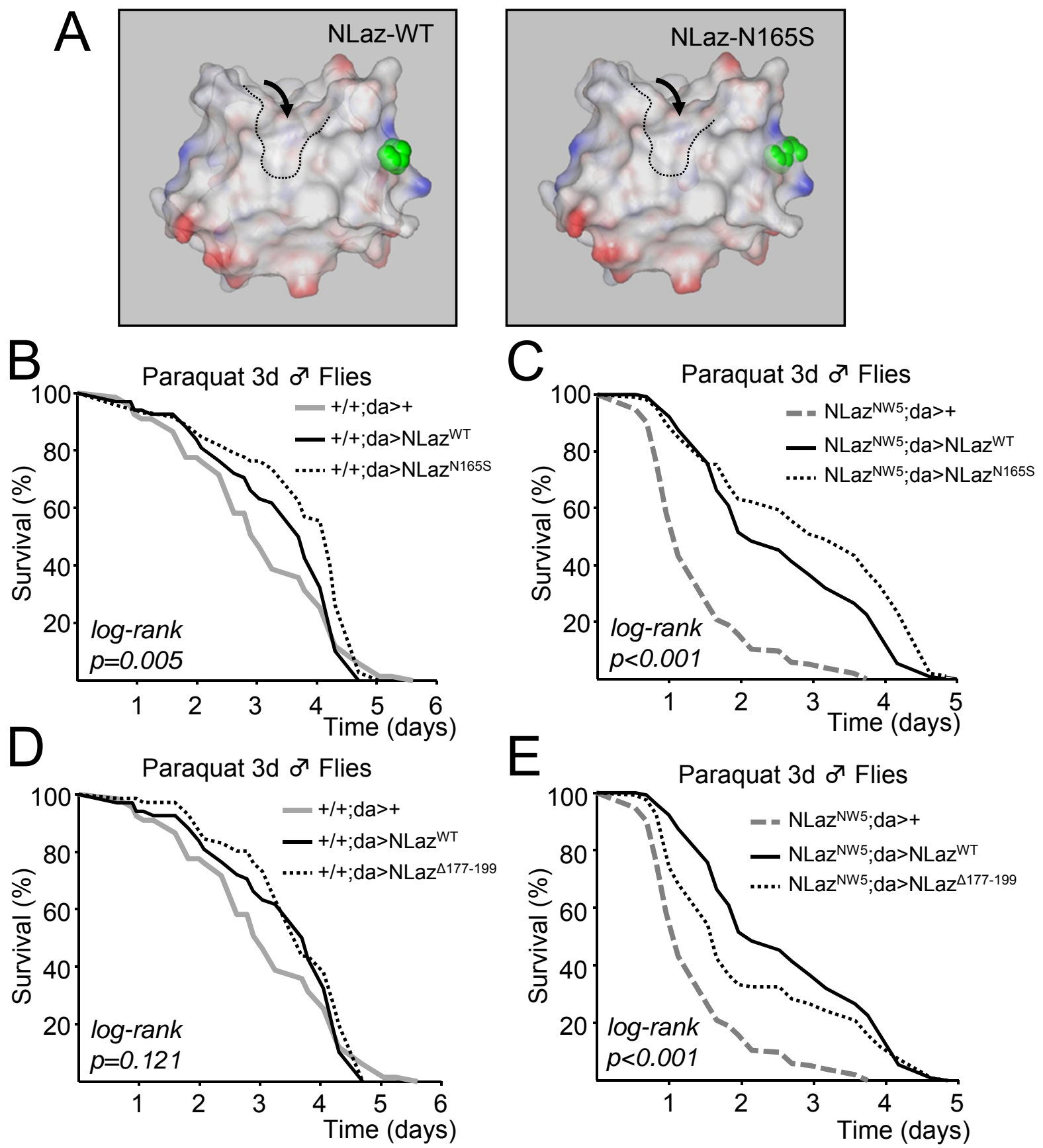

Supplementary Figure 2. Deletion of the C-terminal portion (177-199) of NLaz and the NLaz ${ }^{\mathrm{N} 165 \mathrm{~s}}$ variant alter stress resistance.

(A) Model of NLaz ${ }^{\mathrm{N} 165 \mathrm{~S}}$ protein structure compared to $\mathrm{NLaz}^{\mathrm{WT}}$. Backbone and surface models are superimposed. A lateral view is shown. The binding pocket is pointed by an arrow and is outlined by a dashed line. N165 or S165 residues are labeled in green.

(B) Over-expression of NLaz ${ }^{\mathrm{N} 165 \mathrm{~S}}$ in a wild-type genetic background is able to increase fly resistance against PQ when compared to control flies at 3 days of age.

(C) Resistance to paraquat of young flies expressing NLaz ${ }^{\mathrm{N} 165 \mathrm{~S}}$ in $\mathrm{NLaz}^{\mathrm{NW} 5}$ null background is higher than that of flies expressing $\mathrm{NLaz}^{\mathrm{WT}}$.

(D) Ubiquitous over-expression of both $\mathrm{NLaz}^{\mathrm{WT}}$ and $\mathrm{NLaz}^{\Delta 177-199}$ in wild-type background does not increase significantly the resistance to PQ in young flies.

(E) NLaz ${ }^{\Delta 177-199}$ expression rescues the PQ phenotype when expressed in NLaz ${ }^{\mathrm{NW} 5}$ null mutant background.

Log-rank tests followed by Holm-Sidak multiple comparison post-hoc tests were used in B-E. 
Supplementary Data File 1. Transcriptional profile data. Genes selected for expression studies and gene expression in basal conditions.

Transcriptional profile data. Tables $S 1$ and $S 2$.

\begin{tabular}{|c|c|c|c|c|}
\hline Gene Symbol & Refseq\# & Official Name / Mamalian orthologue & RT2 Cat.No. & Functional category \\
\hline Act5C & NM_001014725 & Actin 5C & $\begin{array}{l}\text { PPD00467 } \\
\end{array}$ & Housekeeping \\
\hline Akt1 & NM_169705 & serine/threonine-protein kinase & PPD09200 & Metabolism control (IIS pathway) \\
\hline bsk & NM_164900 & Basket / JNK & PPD11004 & Stress response pathway (JNK pathway) \\
\hline Cat & NM_080483 & Catalase & PPD07567 & Oxidative stress response (Foxo target) \\
\hline CG12374 & NM 136975 & Carboxypeptidase-like (Zn) CG12374 & PPD04510 & Age/Oxiative stress specific response (vs. starvation/infection) \\
\hline CG8147 & NM_001038951 & Alkaline phosphatase-like CG8147 & PPD08516 & Age/Oxiative stress specific response (vs. starvation/infection) \\
\hline Ddc & NM_078876 & Dopa decarboxylase & PPD03531 & Neurotransmitter/Hormonal systems \\
\hline desat1 & NM_144474 & Desaturase 1 & PPD11642 & Lipid / Hormonal systems \\
\hline Dpt & NM_057460 & Diptericin & PPD05159 & Immune response \\
\hline Drs & NM_079177 & Drosomycin & PPD06186 & Immune response \\
\hline foxo & $\mathrm{NM}_{-}^{-} 142073$ & Forkhead box, sub-group $O$ & PPD08986 & Metabolism control (IIS and Sir2 pathways) \\
\hline Gadd45 & NM_136420 & Growth arrest and DNA damage-inducible & PPD03876 & Oxidative stress response-DNA repair (Foxo target) \\
\hline Gapdh1 & NM_001038847 & Glyceraldehyde 3 phosphate dehydrogenase 1 & PPD03944 & Housekeeping \\
\hline GLaz & NM_079003 & Glial Lazarillo & PPD04541 & Lipocalin family / Oxidative stress response \\
\hline Gr66a & NM_079247 & Gustatory receptor $66 \mathrm{a}$ & PPD06616 & Chemoreception in pheromone sensing neurons \\
\hline GstE1 & NM_137479 & Glutathione $\mathrm{S}$ transferase $\mathrm{E} 1$ & PPD05094 & Oxidative stress response \\
\hline GstS1 & NM_079043 & Glutathione S transferase S1 & PPD66725 & Oxidative stress response \\
\hline Hr96 & NM_079769 & Hormone receptor-like in 96 & PPD10079 & Lipid homeostasis \\
\hline Hsp68 & NM_001031945 & 5 Heat shock gene 68 & PPD13069 & Oxidative stress response (JNK pathway) \\
\hline Ilp4 & NM_140104 & Insulin-like peptide 4 & PPD06800 & Metabolism control / Neurogenesis control (IIS pathway) \\
\hline InR & NM_079712 & Insulin-like receptor & PPD09691 & Metabolism control (IIS pathway) \\
\hline Jafrac1 & NM_058162 & Thioredoxin peroxidase 1 & PPD11559 & Oxidative stress response (Foxo target) \\
\hline Karl & NM_132520 & Karl & PPD00966 & Lipocalin family / Immune response \\
\hline Lip4 & NM_135574 & Lipase 4 & PPD02897 & Metabolism control (Foxo target) \\
\hline Lsd-1 & NM_142926 & Lipid storage droplet-1 & PPD09917 & Lipid metabolism \\
\hline Lsp2 & NM 080077 & Larval serum protein 2 & PPD1 1062 & Lifespan-Aging modulation \\
\hline Mgstl & NM_079957 & Microsomal glutathione S-transferase-like & PPD10883 & Oxidative stress response \\
\hline mio & NM_134812 & Missing oocyte & PPD02026 & Lipid metabolism \\
\hline Nplp3 & NM_144453 & Neuropeptide-like precursor 3 & PPD11605 & Neural activity/ Lifespan-Aging modulation \\
\hline Pepck & NM_079060 & Phosphoenolpyruvate carboxykinase & PPD05115 & Carbohydrate metabolism (Foxo target) \\
\hline Prx2540-2 & NM_078959 & Peroxiredoxin $2540-2$ & PPD04249 & Oxidative stress response \\
\hline puc & NM_079549 & Puckered (JNK-specific phosphatase) & PPD08362 & Stress response pathway (JNK pathway) \\
\hline RpL18 & NM_139834 & Ribosomal protein L18 & PPD06501 & Housekeeping \\
\hline RpL32 & NM_-079843 & Ribosomal protein L32 & PPD10569 & Housekeeping \\
\hline S6k & NM_-079217 & RPS6-p70-protein kinase & PPD06382 & Metabolism control pathway \\
\hline Sir2 & NM_-058003 & NAD-dependent histone deacetylase Sir2 & PPD03110 & Lifespan-Aging modulation / Metabolism control \\
\hline SNPF & NM_-078881 & Short neuropeptide F precursor & PPD03613 & Neuropeptide hormones / Food intake regulation / Stress response \\
\hline Sod2 & NM_-057577 & Superoxide dismutase 2 (Mn-SOD) & PPD04905 & Oxidative stress response \\
\hline Spt-I & NM_136998 & Serine palmitoyltransferase subunit I & PPD04542 & Lipid metabolism \\
\hline synaptogyrin & NM_137064 & Synaptogyrin & PPD04615 & Neural activity/Lifespan-Aging modulation \\
\hline Thor & NM_057947 & Thor / 4E-BP (eIF4E binding protein) & PPD02171 & Metabolism control (IIS - TOR pathways) \\
\hline Tk & NM_141884 & Tachykinin & PPD08785 & Neuropeptide hormones / Stress response \\
\hline to & NM_079773 & Takeout & PPD10117 & Lipid metabolism / Lifespan-Aging modulation \\
\hline whd & NM_078961 & Withered & PPD04258 & Lipid metabolism \\
\hline \multicolumn{5}{|l|}{ Controls } \\
\hline DGDC & SA 00146 & Fly Genomic DNA Contamination & PPD66893 & Technical control \\
\hline RTC & SA_00104 & Reverse Transcription Control & PPX63340 & Technical control \\
\hline PPC & SA 00103 & Positive PCR Control & PPX63339 & Technical control \\
\hline
\end{tabular}

Table S2. Gene expression comparisons in basal conditions. Expression in flies of each genotype at 3 days of age was compared with the w1118; NLaz NW5/+; da:Gal4/+ control. p values are calculated based on a Student's t-test of the replicate 2- $\Delta \mathrm{Ct}$ values for each gene in the control group and treatment groups. p values less than 0.05 are indicated in red. Up-regulations are shown in red and down-reglations in blue.

\begin{tabular}{|c|c|c|c|c|c|c|}
\hline & \multicolumn{2}{|c|}{$-/-; \mathbf{d a}>+$} & \multicolumn{2}{|c|}{$-/-;$ da $>$ Nlaz-WT } & \multicolumn{2}{|c|}{$-/-;$ da $>$ Nlaz-L130R } \\
\hline Gene Symbol & \begin{tabular}{|l|} 
Fold Regulation \\
\end{tabular} & $p$-value & \begin{tabular}{|l|} 
Fold Regulation \\
\end{tabular} & $p$-value & old Regulatio & $p$-valu \\
\hline Act5C & 1.001 & 0.96843 & \begin{tabular}{|l|}
1.289 \\
\end{tabular} & 0.08226 & \begin{tabular}{|l|}
1.281 \\
\end{tabular} & 0.0637 \\
\hline Akt1 & -1.172 & 0.03538 & -1.298 & 0.00568 & -1.447 & 0.0046 \\
\hline bsk & -1.011 & 0.92925 & -1.069 & 0.71340 & -1.032 & $0.6924 \mathrm{C}$ \\
\hline Cat & -1.132 & 0.37087 & -1.057 & 0.56660 & 1.145 & 0.4565 \\
\hline CG1237 & -1.038 & 0.45605 & -1.534 & 0.00009 & -2.228 & $0.0000_{2}$ \\
\hline CG8147 & -1.483 & 0.00259 & -10.367 & 0.00000 & -3.809 & 0.0000 \\
\hline Ddc & 1.008 & 0.93340 & 1.047 & 0.68979 & 1.168 & 0.14218 \\
\hline desat1 & -1.051 & 0.46832 & -1.243 & 0.04395 & -1.078 & 0.2714 \\
\hline Dpt & 4.410 & 0.00002 & 63.570 & 0.00000 & 13.513 & 0.0000 \\
\hline Drs & -1.113 & 0.65387 & 4.599 & 0.00004 & 1.456 & 0.0075 \\
\hline foxo & -1.345 & 0.00976 & -1.313 & 0.00438 & -1.450 & 0.0015 \\
\hline Gadd45 & -1.307 & 0.12868 & -1.220 & 0.13537 & -1.060 & 0.7258 \\
\hline Gapdh1 & -1.352 & 0.19108 & -1.425 & 0.05864 & -1.303 & 0.0728 \\
\hline GLaz & 1.009 & 0.87518 & -1.082 & 0.10049 & -1.263 & 0.0078 \\
\hline Gr66a & -1.340 & 0.33279 & -1.177 & 0.49413 & 1.247 & 0.4709 \\
\hline GstEl & -1.191 & 0.68953 & 1.382 & 0.20157 & 1.241 & 0.2928 \\
\hline GstS1 & -1.301 & 0.00370 & -1.962 & 0.00010 & -1.838 & 0.0003 \\
\hline $\mathrm{Hr} 96$ & -1.076 & 0.48599 & -1.295 & 0.02979 & -1.015 & 0.83008 \\
\hline Hsp68 & -1.268 & 0.00516 & -1.203 & 0.02425 & \begin{tabular}{|c|}
-1.371 \\
\end{tabular} & 0.0022 \\
\hline $\begin{array}{ll}\operatorname{InR} \\
\end{array}$ & -1.586 & 0.00060 & -1.929 & 0.00016 & -1.383 & 0.0168 \\
\hline Jafrac & 1.101 & 0.19687 & 1.079 & 0.14682 & 1.022 & 0.7670 \\
\hline Karl & -2.434 & 0.00022 & 2.674 & 0.00000 & 1.026 & 0.7716 \\
\hline Lip4 & 1.311 & 0.02100 & -1.253 & 0.02485 & -1.203 & 0.0696 \\
\hline $11 p 4$ & -4.167 & 0.02306 & -4.076 & 0.03266 & -3.060 & 0.0401 \\
\hline Lsd-1 & -1.271 & 0.00975 & -1.992 & 0.00001 & -1.618 & 0.0001 \\
\hline Lsp2 & 1.088 & 0.56817 & 2.084 & 0.00010 & -1.009 & 0.8873 \\
\hline Mgstl & -1.023 & 0.89408 & 1.203 & 0.17277 & 1.254 & 0.0905 \\
\hline mio & -1.895 & 0.03709 & -2.085 & 0.0640 & -2.276 & 0.0499. \\
\hline Nplp3 & -1.825 & 0.00030 & -2.665 & 0.00002 & -2.892 & 0.0000 \\
\hline Pepck & -1.624 & 0.00040 & 1.115 & 0.08810 & 1.276 & 0.0058 \\
\hline Prx254 & $\begin{array}{l}1.049 \\
\end{array}$ & 0.54468 & -1.223 & 0.03380 & 1.150 & 0.0919 \\
\hline puc & -1.142 & 0.03390 & -1.366 & 0.02986 & -1.132 & 0.3722 \\
\hline RpL18 & 1.160 & 0.55173 & 1.392 & 0.14750 & 1.757 & 0.0080 \\
\hline RpL32 & 1.000 & 1.00000 & 1.000 & 1.00000 & 1.000 & 1.0000 \\
\hline S6k & -1.372 & 0.04942 & -1.623 & 0.00530 & -1.499 & 0.0234 \\
\hline Sir2 & 1.050 & 0.60638 & -1.093 & 0.17760 & -1.019 & 0.7228 \\
\hline sNPF & -1.154 & 0.37812 & -1.115 & 0.33652 & -1.154 & 0.2205 \\
\hline Sod2 & -1.129 & 0.37138 & -1.099 & 0.33513 & -1.122 & 0.3533 \\
\hline $\begin{array}{lll}\text { Spt-1 } \\
\end{array}$ & 1.262 & 0.02655 & 1.009 & 0.90201 & -1.075 & 0.4187 \\
\hline synapt & -1.226 & 0.00053 & -1.169 & 0.00209 & -1.163 & 0.2041 \\
\hline Thor & -1.277 & 0.00136 & -1.354 & 0.00163 & 1.154 & 0.0294 \\
\hline $\begin{array}{ll}\mathrm{Tk} \\
\end{array}$ & -1.003 & 0.87159 & -1.880 & 0.01431 & -1.525 & 0.0750 \\
\hline to & -1.221 & 0.04269 & -1.030 & 0.54535 & -1.349 & 0.0001. \\
\hline whd & -1.473 & 0.00002 & -1.579 & 0.00004 & -1.353 & 0.0366 \\
\hline
\end{tabular}


Table S3. Gene expression comparisons of 3 days flies treated for $17 \mathrm{~h}$ with PQ with control flies of each genotype. $p$ values are calculated based on a Student's t-test of the replicate $2^{-\Delta C t}$ values for each gene in the control group and treatment groups. $p$ values less than 0.05 are indicated in red. Up-regulations are shown in red and down-regulations in blue.

\begin{tabular}{|c|c|c|c|c|c|c|c|c|}
\hline \multirow[b]{2}{*}{ Gene Symbol } & \multicolumn{2}{|c|}{+ +/-; da>+(PQ vs. Control) } & \multicolumn{2}{|c|}{ 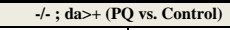 } & \multicolumn{2}{|c|}{$-/-;$ da $>$ Nlaz-WT (PQ vs. Control) } & \multicolumn{2}{|c|}{$-1-;$ da $>$ Nlaz-L130R (PQ vs. Control) } \\
\hline & Fold Regulation & p-value & Fold Regulation & p-value & Fold Regulation & p-value & Fold Regulation & $p$-value \\
\hline \begin{tabular}{l|} 
Act5C \\
\end{tabular} & 1.080 & 0.64586 & 1.084 & 0.67628 & -1.137 & 0.14188 & 1.567 & 0.00000 \\
\hline Akt1 & 1.017 & 0.82186 & 1.450 & 0.00007 & 1.380 & 0.00123 & 1.571 & 0.00027 \\
\hline bsk & -1.349 & 0.22559 & 1.046 & 0.86969 & 1.143 & 0.44734 & 1.279 & 0.03281 \\
\hline Cat & -1.039 & 0.76239 & 1.556 & 0.00022 & 1.183 & 0.00295 & 1.524 & 0.01534 \\
\hline CG1237 & -9.970 & 0.00000 & -13.674 & 0.00000 & -7.387 & 0.00000 & -5.051 & 0.00000 \\
\hline CG8147 & -27.606 & 0.00000 & -44.839 & 0.00000 & -5.805 & 0.00000 & -13.511 & 0.00000 \\
\hline $\mathrm{Ddc}$ & -1.516 & 0.01410 & -1.337 & 0.02769 & -1.354 & 0.00493 & 1.056 & 0.32784 \\
\hline desatl & -2.420 & 0.00010 & -2.027 & 0.00001 & -1.600 & 0.00248 & -1.396 & 0.00021 \\
\hline Dpt & 2.255 & 0.00004 & 1.991 & 0.00004 & -20.740 & 0.00000 & -2.257 & 0.00008 \\
\hline Drs & 1.812 & 0.02333 & 2.340 & 0.00036 & -2.473 & 0.00026 & 1.512 & 0.00036 \\
\hline foxo & -1.774 & 0.00019 & -1.251 & 0.01420 & $\begin{array}{l}-1.442 \\
\end{array}$ & 0.00010 & -1.072 & 0.16082 \\
\hline Gadd45 & 3.419 & $\begin{array}{l}0.00037 \\
\end{array}$ & 7.415 & 0.00000 & 2.620 & 0.00000 & $\begin{array}{l}4.614 \\
\end{array}$ & 0.00001 \\
\hline Gapdh1 & -3.602 & 0.00342 & -1.567 & 0.07232 & -2.174 & 0.00263 & -1.045 & 0.44976 \\
\hline GLaz & -2.141 & 0.00002 & -2.221 & 0.00000 & -1.667 & 0.00000 & -1.181 & 0.04135 \\
\hline $\begin{array}{l}\text { Gr66a } \\
\end{array}$ & -1.025 & 0.88273 & 2.954 & 0.00068 & 1.821 & 0.00593 & 1.535 & 0.01218 \\
\hline GstE1 & 10.947 & 0.00440 & 16.078 & 0.00007 & 6.604 & 0.00080 & 16.503 & 0.00000 \\
\hline GstS1 & -1.759 & 0.00054 & -1.230 & 0.00161 & 1.028 & 0.74450 & 1.217 & 0.10148 \\
\hline $\mathrm{Hr} 96$ & -1.127 & 0.16779 & -3.406 & 0.37900 & 1.063 & 0.54653 & 1.172 & 0.01844 \\
\hline Hsp68 & 1.399 & 0.00282 & 5.755 & 0.00000 & 2.288 & 0.00001 & 1.732 & 0.00000 \\
\hline InR & 1.897 & 0.00025 & 4.467 & 0.00000 & 3.141 & 0.00091 & 2.096 & 0.00084 \\
\hline Jafrac & 1.425 & 0.00138 & 1.278 & 0.00169 & 1.183 & 0.00266 & 2.353 & 0.00001 \\
\hline Karl & -1.894 & 0.00325 & 1.347 & 0.02570 & -1.951 & 0.00005 & 1.719 & 0.00298 \\
\hline Lip4 & 2.035 & 0.00002 & 1.002 & 0.96271 & 2.575 & 0.00000 & 3.274 & 0.00000 \\
\hline 11p4 & -3.488 & 0.03697 & 2.117 & 0.18010 & -1.140 & 0.58492 & 1.010 & 0.93531 \\
\hline Lsd-1 & -1.962 & 0.00002 & -2.408 & 0.00005 & -1.036 & 0.27274 & 1.496 & 0.00007 \\
\hline Lsp2 & -2.490 & 0.00216 & 1.346 & 0.00192 & -3.066 & 0.00000 & 1.225 & 0.06080 \\
\hline Mgstl & -1.434 & 0.05709 & -1.209 & 0.15701 & -1.551 & 0.00013 & 1.107 & 0.01213 \\
\hline mio & 1.025 & 0.96199 & 2.531 & 0.00023 & -1.079 & 0.79606 & 2.002 & 0.16243 \\
\hline Nplp3 & \begin{tabular}{|l|}
-3.814 \\
\end{tabular} & 0.00001 & -4.428 & 0.00002 & $\begin{array}{l}-6.671 \\
\end{array}$ & 0.00000 & -1.409 & 0.02443 \\
\hline Pepck & 2.379 & 0.00021 & 4.731 & 0.00000 & 3.471 & 0.00002 & 2.212 & 0.00000 \\
\hline Prx254 & 2.950 & 0.00000 & 2.509 & 0.00001 & 1.732 & 0.00012 & 1.245 & 0.00331 \\
\hline puc & 1.371 & 0.00356 & 2.508 & 0.00001 & 1.271 & 0.23545 & 1.576 & 0.09594 \\
\hline RpL18 & 1.146 & 0.58380 & 1.026 & 0.91643 & 1.106 & 0.51049 & 1.432 & 0.00059 \\
\hline RpL32 & 1.000 & 1.00000 & 1.000 & 1.00000 & 1.000 & 1.00000 & 1.000 & 1.00000 \\
\hline S6k & -1.581 & 0.01124 & -1.090 & 0.48048 & -1.149 & 0.32823 & 1.051 & 0.74253 \\
\hline Sir2 & -1.113 & 0.27683 & 1.311 & 0.01832 & 1.203 & 0.01185 & 1.151 & 0.00934 \\
\hline sNPF & -1.148 & 0.22731 & 1.291 & 0.09971 & 1.122 & 0.18456 & 1.235 & 0.00856 \\
\hline Sod2 & -1.663 & 0.00942 & -1.314 & 0.02937 & -1.523 & 0.00000 & 1.064 & 0.46572 \\
\hline Spt-1 & -1.404 & 0.00161 & -1.628 & 0.00102 & -1.245 & 0.00952 & -1.080 & 0.36353 \\
\hline synapt & -1.571 & 0.00005 & -1.012 & 0.81790 & -1.014 & 0.86483 & 1.034 & 0.85412 \\
\hline Thor & 2.277 & 0.00007 & 4.880 & 0.00000 & 2.923 & 0.00001 & 2.299 & 0.00000 \\
\hline $\mathrm{Tk}$ & -1.561 & 0.04728 & -1.402 & 0.00588 & 1.484 & 0.01579 & 1.383 & 0.06986 \\
\hline to & -1.183 & 0.08093 & 1.092 & 0.36494 & 1.318 & 0.00477 & 1.476 & 0.00005 \\
\hline whd & -1.184 & 0.00271 & 1.270 & 0.00164 & 1.200 & 0.00603 & 1.115 & 0.53095 \\
\hline
\end{tabular}

Table S4. Gene expression comparisons of 3 vs. 30 day-old flies of each genotype. $p$ values are calculated based on a Student's t-test of the replicate $2^{-\Delta C t}$ values for each gene in the control group and treatment groups. $p$ values less than 0.05 are indicated in red. Up-regulations are shown in red and down-regulations in blue.

\begin{tabular}{|c|c|c|c|c|c|c|c|c|}
\hline & \multicolumn{2}{|c|}{\begin{tabular}{|l}
$+-; \mathbf{d a}>+(30$ d vs. 3 d) \\
\end{tabular}} & \multicolumn{2}{|c|}{$-/-;$ da $>+(30$ d vs. 3 d $)$} & \multicolumn{2}{|c|}{$-/$; da $>$ Nlaz-WT (30 d vs. 3 d) } & \multicolumn{2}{|c|}{$-1-;$ da $>$ Nlaz-L130R (30 d vs. 3 d) } \\
\hline Gene Symbol & \begin{tabular}{|l|} 
Fold Regulation \\
\end{tabular} & p-value & \begin{tabular}{|l|} 
Fold Regulation \\
\end{tabular} & p-value & Fold Regulation & p-value & Fold Regulation & p-value \\
\hline Act5C & -1.542 & 0.06321 & 1.197 & 0.29363 & \begin{tabular}{|l|l}
-1.401 \\
\end{tabular} & 0.02413 & 2.156 & 0.00001 \\
\hline Akt1 & -1.672 & 0.00040 & 1.625 & 0.00192 & -1.183 & 0.02144 & 1.806 & 0.00007 \\
\hline bsk & -1.837 & 0.02556 & 1.376 & 0.07002 & -1.777 & 0.06654 & 1.875 & 0.00001 \\
\hline Cat & -1.140 & 0.59566 & 2.002 & 0.00058 & 1.043 & 0.64200 & 1.371 & 0.04269 \\
\hline CG1237 & -4.017 & 0.00000 & -13.712 & 0.00000 & -3.657 & 0.00000 & -7.343 & 0.00000 \\
\hline CG8147 & -8.803 & 0.00000 & -22.907 & 0.00000 & 2.798 & 0.00001 & -1.316 & 0.00001 \\
\hline Ddc & -1.995 & 0.00307 & 1.068 & 0.58010 & -1.713 & 0.00064 & 1.256 & 0.02046 \\
\hline desat 1 & -1.288 & 0.02715 & -1.771 & 0.00094 & 1.444 & 0.00859 & -1.115 & 0.05137 \\
\hline Dpt & 1.171 & 0.23333 & -1.130 & 0.22003 & -3.122 & 0.00000 & 42.877 & 0.00000 \\
\hline Drs & -1.228 & 0.38725 & 2.511 & 0.00032 & -4.091 & 0.00006 & 14.817 & 0.00000 \\
\hline foxo & -1.542 & 0.00102 & -1.159 & 0.33855 & -1.211 & 0.02149 & 1.387 & 0.00010 \\
\hline Gadd45 & -2.492 & 0.00519 & 13.009 & 0.00000 & -1.167 & 0.08599 & 2.936 & 0.00001 \\
\hline Gapdh1 & -9.964 & 0.00018 & 1.433 & 0.08215 & -5.559 & 0.00020 & -3.726 & 0.00000 \\
\hline GLaz & -1.743 & 0.00095 & -1.909 & 0.00002 & -1.544 & 0.00000 & 1.054 & 0.37975 \\
\hline Gr66a & -1.385 & 0.36476 & 5.332 & 0.00015 & 1.324 & 0.20643 & 2.294 & 0.00013 \\
\hline GstE1 & -1.217 & 0.80575 & 26.472 & 0.00006 & $\begin{array}{l}1.313 \\
\end{array}$ & 0.14101 & 4.203 & 0.00000 \\
\hline GstS1 & -4.133 & 0.00002 & -1.053 & 0.49674 & -2.662 & 0.00364 & -1.360 & 0.01648 \\
\hline $\begin{array}{ll}\mathrm{Hr} 96 \\
\end{array}$ & -1.678 & 0.00351 & 1.214 & 0.15648 & -1.093 & 0.21807 & 1.335 & 0.00199 \\
\hline Hsp68 & -2.046 & 0.00005 & 6.524 & 0.00002 & \begin{tabular}{|l|l}
-1.970 \\
\end{tabular} & 0.00004 & 2.660 & 0.00000 \\
\hline InR & -2.084 & 0.00230 & 4.690 & 0.00011 & 1.042 & 0.59538 & 2.500 & 0.00004 \\
\hline Jafrac & -1.330 & 0.01034 & 1.632 & 0.00333 & -1.189 & 0.00028 & 1.491 & 0.00054 \\
\hline $\begin{array}{l}\text { Karl } \\
\end{array}$ & -4.532 & 0.00007 & 1.622 & 0.01491 & -1.255 & 0.00156 & 1.960 & 0.00002 \\
\hline Lip4 & -1.181 & 0.12714 & 1.067 & 0.51384 & 1.045 & 0.10813 & 1.686 & 0.00004 \\
\hline 11p4 & -4.712 & 0.02160 & 3.039 & 0.02089 & -3.311 & 0.29345 & 1.306 & 0.46516 \\
\hline Lsd-1 & -1.848 & 0.00003 & -1.924 & 0.00016 & 1.934 & 0.00087 & 1.004 & 0.94163 \\
\hline Lsp2 & 1.167 & 0.32893 & 1.717 & 0.00036 & 3.499 & 0.00000 & 2.975 & 0.00001 \\
\hline Mgstl & -1.816 & 0.01465 & -1.060 & 0.55326 & -1.362 & 0.01045 & -1.145 & 0.01262 \\
\hline mio & -2.228 & 0.02669 & 2.815 & 0.00309 & -2.186 & 0.52800 & 2.559 & 0.00214 \\
\hline Nplp3 & -28.416 & 0.00000 & -1.792 & 0.00253 & -10.340 & 0.00000 & -3.613 & 0.00027 \\
\hline Pepck & 1.936 & 0.00003 & 5.351 & 0.00002 & 2.970 & 0.00011 & 1.335 & 0.00043 \\
\hline Prx254 & -2.002 & 0.00019 & 3.876 & 0.00011 & -1.912 & 0.00001 & -1.184 & 0.00754 \\
\hline puc & -1.840 & 0.00880 & 3.589 & 0.00002 & -1.854 & 0.65336 & 3.579 & 0.00000 \\
\hline RpL18 & 1.142 & 0.55893 & 1.465 & 0.12492 & 1.546 & 0.01499 & 1.307 & 0.01551 \\
\hline RpL32 & 1.000 & 1.00000 & 1.000 & 1.00000 & 1.000 & 1.00000 & 1.000 & 1.00000 \\
\hline S6k & -1.825 & 0.00455 & 1.061 & 0.68089 & -1.090 & 0.17141 & 1.473 & 0.00468 \\
\hline $\begin{array}{l}\text { Sir2 } \\
\end{array}$ & -1.373 & 0.00288 & 1.927 & 0.00048 & -1.024 & 0.70739 & 1.790 & 0.00002 \\
\hline sNPF & -1.473 & 0.08927 & 1.492 & 0.01589 & -1.206 & 0.10719 & 2.058 & 0.00002 \\
\hline Sod2 & -3.013 & 0.00072 & 1.194 & 0.13122 & -1.899 & 0.00000 & -1.563 & 0.00231 \\
\hline Spt-1 & -1.284 & 0.03626 & -1.303 & 0.05097 & -1.448 & 0.00049 & 1.355 & 0.00208 \\
\hline synapt & -3.063 & 0.00000 & 1.473 & 0.00262 & -2.000 & 0.00001 & 1.018 & 0.96504 \\
\hline Thor & -2.229 & 0.00001 & 6.340 & 0.00002 & 1.088 & 0.14582 & 1.326 & 0.00044 \\
\hline $\mathrm{Tk}$ & -2.567 & 0.00561 & -1.181 & 0.06543 & -1.490 & 0.04258 & 1.233 & 0.24832 \\
\hline to & -1.403 & 0.00485 & 1.492 & 0.00536 & 1.641 & 0.00029 & 2.100 & 0.00000 \\
\hline whd & -1.503 & 0.00081 & 1.548 & 0.00278 & 1.068 & 0.57877 & 1.382 & 0.02675 \\
\hline
\end{tabular}

